\title{
MYBL2-induced PITPNA-AS1 upregulates SIK2 to exert oncogenic function in triple-negative breast cancer through miR-520d-5p and DDX54
}

\author{
Bolong Liu', Pingbo Yao ${ }^{2}$, Feng Xiao ${ }^{3}$, Jianjin Guo ${ }^{4}$, Lianghui $\mathrm{Wu}^{5^{*}}$ and Yong Yang ${ }^{6^{*}}$
}

\begin{abstract}
Background: In recent years, long non-coding RNAs (IncRNAs) have attracted much attention because of its regulatory role in occurrence and progression of tumors, including triple-negative breast cancer (TNBC). LnCRNA PITPNA antisense RNA 1 (PITPNA-AS1) has been explored in some cancers, whereas its function and molecular mechanism in TNBC remain unclear.

Methods: PITPNA-AS1 expression in TNBC tissues and cells was determined by RT-qPCR. TNBC cell viability, proliferation, migration, invasion were assessed with CCK-8, colony formation, wound healing, transwell assays. Cell apoptosis was evaluated by flow cytometry. Expression of EMT-related markers was detected by western blot analyses. The molecular mechanism of PITPNA-AS1 was explored by RNA pull down, luciferase reporter, RIP and ChIP assays.

Results: PITPNA-AS1 showed high expression levels in TNBC tissues and cells. PITPNA-AS1 knockdown suppressed TNBC cell viability, proliferation, migration, invasion in vitro and inhibited xenograft tumor growth in mice. Mechanistically, PITPNA-AS1 upregulated SIK2 expression by sponging miR-520d-5p and recruiting DDX54 protein. Results of rescue assays suggested that the inhibitive effects of silenced PITPNA-AS1 on TNBC cellular processes were partially rescued by overexpressing SIK2 or combination of miR-520d-5p inhibition and DDX54 overexpression. More importantly, we found that the upregulation of PITPNA-AS1 in TNBC cells was attributed to transcription factor MYBL2.
\end{abstract}

Conclusion: PITPNA-AS1 activated by MYBL2 plays an oncogenic role in TNBC through upregulating SIK2.

Keywords: PITPNA-AS1, miR-520d-5p, DDX54, SIK2, TNBC

\section{Introduction}

There is a rapid rising trend in the incidence of breast cancer $(\mathrm{BC})$ with more than 1 million new-diagnosed

\footnotetext{
*Correspondence: wulianghui20051217@163.com; maji1177@sina.com

${ }^{\dagger}$ Bolong Liu and Pingbo Yao contributed equally to this work

${ }^{5}$ Department of Intensive Care Unit, Affiliated Nanhua Hospital,

University of South China, No. 336, Dongfeng South Road, Zhuhui District,

Hengyang 421001, Hunan, China

${ }^{6}$ Department of General Surgery, The Second Hospital, University

of South China, 30 Jiefang Road, Shigu District, Hengyang 421001, Hunan, China

Full list of author information is available at the end of the article
}

cases each year [1]. According to molecular characteristics of HER-2, estrogen receptor (ER), ki-67, and progesterone receptor (PR), $\mathrm{BC}$ can be divided into diverse subtypes, such as HER-2 overexpression, Lumina A, Lumina B, 'normal-like' breast tumors and basallike tumors. As defined, triple-negative breast cancer (TNBC) is a tumor that is negative for HER-2, ER and PR [2]. Currently, surgical resection and chemotherapy are the major systemic therapies for patients with TNBC due to the lack of effective biomarker [3]. Compared with patients with other BC subtypes, TNBC patients have 
significantly higher rates of metastasis and recurrence $[4$, 5]. Thus, it is essential to explore more effective biomarkers for early diagnosis and advanced treatment of TNBC.

It was commonly accepted that non-coding RNAs (ncRNAs) act as critical factors in the regulation of gene expression, thereby exerting functions on tumor or nontumor cell phenotypes [6,7]. Long non-coding RNAs (lncRNAs) are generally regarded as genomic transcripts exceeding 200 nucleotides in length [8]. Lacking open reading structure of indispensable length, lncRNAs are limited in protein-coding [9]. It has been highlighted by diverse researches that lncRNAs are associated with molecular mechanisms underlying cancer development and can be used as promising biomarkers and therapeutic targets for cancers [10]. Previously, many studies have emphasized the important roles of lncRNAs in regulating various cell biological behaviors including cell growth, apoptosis, migration and invasion [11, 12]. Increasing lncRNAs, such as NEAT1 [13], BORG [14] and LINC01638 [15], have been identified as oncogenes in TNBC.

LncRNAs are implicated in tumorigenesis and progression of cancers via multiple mechanisms [16]. Importantly, lncRNA can function as a competing endogenous RNA (ceRNA) to upregulate mRNA expression by sponging microRNA (miRNA). For example, lncRNA EPB41L4A-AS2 upregulates FOXL1 expression to suppress hepatocellular carcinoma progression via sponging miR-301a-5p [17]. LncRNA NORAD facilitates cervical cancer cell proliferation and invasion by targeting the miR-590-3p/SIP1 axis [18]. In addition, IncRNAs can modulate tumor initiation by binding with RNA-binding proteins (RBPs) to maintain mRNA stability. For instance, LINC00324 positively regulates FAM83B mRNA stability to promote cell proliferation in gastric cancer through binding with $\mathrm{HuR}$ protein [19]. LBX2-AS1 stabilizes LBX2 mRNA expression to drive gastric cancer progression via recruiting FUS protein [20]. LncRNA PITPNA antisense RNA 1 (PITPNA-AS1) has been previously reported as an oncogene in hepatocellular carcinoma by acting as a ceRNA [21]. However, its functional role and molecular mechanism in TNBC remain unclear.

In this research, we investigated the function and regulatory mechanism of PITPNA-AS1 in TNBC cellular processes. Our findings revealed that MYBL2-activated PITPNA-AS1 sponged miR-520d-5p and recruited DDX54 protein to increase SIK2 expression, thereby promoting cellular activities in TNBC. This finding implicated PITPNA-AS1 as a promising biomarker for TNBC treatment.

\section{Materials and methods \\ Tissue samples}

Total 56 pairs of TNBC tissues and their adjacent nontumor tissues were collected from patients who were diagnosed with TNBC at the Second Hospital of Shanxi Medical University (Shanxi, China). Before the surgery, all patients have signed informed consents and none of them received any anti-cancer treatments. The specimens collected from the patients were snap-frozen in liquid nitrogen and subsequently preserved at $-80{ }^{\circ} \mathrm{C}$. Gene expression analysis using 56 pairs of TNBC tissues and corresponding non-tumor tissues was conducted, which was approved by Institutional Ethics Committees of the Second Hospital of Shanxi Medical University.

\section{Cell lines}

One normal breast epithelial cell, MCF10A, and four human TNBC cell lines (HCC1937, MDA-MB-468, MDA-MB-231, MDA-MB-436) were bought from ATCC (Manassas, VA). Cells were incubated in DMEM (Invitrogen, Carlsbad, CA) containing 10\% fetal bovine serum (FBS) and $1 \%$ penicillin/streptomycin in a humidified air at $37^{\circ} \mathrm{C}$ with $5 \% \mathrm{CO}_{2}$.

\section{Cell transfection}

HCC1937 and MDA-MB-468 cells were transfected with the sh-RNAs (plasmid backbone: pGPU6/Hygro) targeting PITPNA-AS1 (sh-PITPNA-AS1\#1/2), DDX54 (sh-DDX54\#1/2), MYBL2 (sh-MYBL2) and corresponding negative controls (sh-NC) at the final concentration of $100 \mathrm{nM}$. Stably transfected cells were selected by detection of Hygromycin B resistance. To overexpress SIK2, E2F6, PAX5, FOXP2, ELK5, MYBL2 and YY1, pcDNA3.1 vector targeting corresponding genes and empty pcDNA3.1 vector (control) were transfected into HCC1937 and MDA-MB-468 cells. For overexpressing or silencing miR-520d-5p, miR-520d-5p mimics or inhibitor was transfected into HCC1937 and MDA-MB-468 cells with NC mimics or inhibitor as a negative control, separately. All above plasmids or oligonucleotides were purchased from GenePharma and were transfected into HCC1937 and MDA-MB-468 cells using Lipofectamine 2000 (Invitrogen) for 2 days.

\section{RT-qPCR analysis}

Using TRIzol Reagent (Invitrogen), total RNA was extracted from MCF10A, HCC1937, MDA-MB-468, MDA-MB-231, MDA-MB-436 cells and then reversetranscribed into cDNA with a Reverse Transcription Kit (Takara). A One Step TB Green ${ }^{\circledR}$ PrimeScript $^{\mathrm{TM}}$ RTPCR Kit (Takara) was utilized for RT-qPCR on a BioRad CFX96 Real-Time PCR system. The $2^{-\Delta \Delta C t}$ method [22] was used for calculation of relative expression fold 
changes. GAPDH served as the internal reference for PITPNA-AS1 and mRNAs, while U6 served as the internal reference for miRNAs. The thermo-cycling conditions were set as follows: $95^{\circ} \mathrm{C}$ for 5 min followed by 45 cycles at $95{ }^{\circ} \mathrm{C}$ for $10 \mathrm{~s}$ and $55^{\circ} \mathrm{C}$ for $30 \mathrm{~s}$, and a melting curve analysis every $0.2^{\circ} \mathrm{C}$ from 55 to $95^{\circ} \mathrm{C}$ for 2 min was obtained. Relative primer sequences are listed in Table 1, which were designed using GETprime software and synthesized by RiboBio (Guangzhou, China).

Table 1 Relative primer sequences used for PCR

\begin{tabular}{|c|c|}
\hline Gene & Primer sequences \\
\hline \multirow[t]{2}{*}{ PITPNA-AS1 } & Forward: 5'-GCAGGGTGGATAAAGAGGA-3' \\
\hline & Reverse: 5'-CCTACTGACAGGATGTCCT-3' \\
\hline \multirow[t]{2}{*}{ miR-524-5p } & Forward: 5'-CTACAAAGGGAAGCACTTTCTCG-3' \\
\hline & Reverse: 5'-CTCTACAGCTATATTGCCAGCCAC-3' \\
\hline \multirow[t]{2}{*}{ miR-520d-5p } & Forward: 5'-CTACAAAGGGAAGCCCTTTCG-3' \\
\hline & Reverse: 5'-CTCTACAGCTATATTGCCAGCCAC-3' \\
\hline \multirow[t]{2}{*}{ U6 } & Forward: 5'-CTCGCTTCGGCAGCACA-3' \\
\hline & Reverse: 5'-AACGCTTCACGAATTTGCGT-3' \\
\hline \multirow[t]{2}{*}{ DENND1B } & Forward: 5'-TGCATCCTTAGTTACCTTCCC-3' \\
\hline & Reverse: 5'-CAGTTCCTTAGCCAAGTAATCTG-3' \\
\hline \multirow[t]{2}{*}{ ATL3 } & Forward: 5'-CAAGAGGAGCAGTGTAATGTG-3' \\
\hline & Reverse: 5'-AAACGTTCCATTGCTTCGT-3' \\
\hline \multirow[t]{2}{*}{ SIK2 } & Forward: 5'-GAGGTCCACAACAGGTCTC-3' \\
\hline & Reverse: 5'-TGCTACAATTCCCTGGGTG-3' \\
\hline \multirow[t]{2}{*}{ SNRNP200 } & Forward: 5'-CTCCAATGCCAAGGATGTG-3' \\
\hline & Reverse: 5'-CTGATGTTGAAGCCCTGGA-3' \\
\hline \multirow[t]{2}{*}{ AFF3 } & Forward: 5'-TACAACTTCCACACCAGCA-3' \\
\hline & Reverse: 5'-ATCTCCAAGCAAGGTCCTG-3' \\
\hline \multirow[t]{2}{*}{ UBE2J1 } & Forward: 5'-GTACATCGTACGGACTCCAG-3' \\
\hline & Reverse: 5'-TCATGGAGGTATTCTTAGCTACAG-3' \\
\hline \multirow[t]{2}{*}{ DECR1 } & Forward: 5'-GGTAAAGGAATGACAACTCTTCTG-3' \\
\hline & Reverse: 5'-CATTTCGGCTGGCTATCAC-3' \\
\hline \multirow[t]{2}{*}{ DDX54 } & Forward: 5'-TGGTGAGTGATCGAGTGAG-3' \\
\hline & Reverse: 5'-CTTCCTGTCCTGACTGTCC-3' \\
\hline \multirow[t]{2}{*}{ MYBL2 } & Forward: 5'-TCCTGGATTCCTGTAACAGC-3' \\
\hline & Reverse: 5'-AGTTCAGAAACTGGGAGGG-3' \\
\hline \multirow[t]{2}{*}{ E2F6 } & Forward: 5'-TAGATGGATAGGATCTGATCTTAGC-3' \\
\hline & Reverse: 5'-GATAAGTCAGAAAGTTCCTCCTG-3' \\
\hline \multirow[t]{2}{*}{ PAX5 } & Forward: 5'-TGTTTGCCTGGGAGATCAG-3' \\
\hline & Reverse: 5'-CGGATGATCCTGTTGATGGA-3' \\
\hline \multirow[t]{2}{*}{ FOXP2 } & Forward: 5'-GATGCAACAACTCCAGCAG-3' \\
\hline & Reverse: 5'-AGGACTTAAGCCAGCTTGAG-3' \\
\hline \multirow[t]{2}{*}{ ELK4 } & Forward: 5'-GCCCACTGGGAATACTGAG-3' \\
\hline & Reverse: 5'-TCAGTATGATGGGTGTCTGTG-3' \\
\hline \multirow[t]{2}{*}{ YY1 } & Forward: 5'-GAATTTGCTAGGGCTGCAC-3' \\
\hline & Reverse: 5'-CACATTCTGCACAGACGTG-3' \\
\hline \multirow[t]{2}{*}{ GAPDH } & Forward: 5'-GCATCCTGGGCTACACTG-3' \\
\hline & Reverse: 5'-TGGTCGTTGAGGGCAAT-3' \\
\hline
\end{tabular}

\section{Subcellular fractionation assay}

Subcellular fractionation assay was conducted to assess subcellular localization of PITPNA-AS1. According to manufacturer' instructions, nuclear and cytoplasmic fractions extracted from TNBC cells were isolated using a Cytoplasmic and Nuclear RNA Purification Kit (Norgen). RT-qPCR analysis was applied for the detection of PITPNA-AS1, GAPDH or U6 expression. GAPDH was a cytoplasmic control and U6 was a nuclear control.

\section{Fluorescence in situ hybridization (FISH) assay}

FISH assay was conducted for detection of the subcellular location of PITPNA-AS1. HCC1937 and MDA-MB-468 cells $\left(5 \times 10^{3}\right.$ cells per well) were cultured in a 24-well plate, and the supernatant was discarded after $24 \mathrm{~h}$. After being washed with PBS and fixed by $4 \%$ paraformaldehyde, the cells were permeabilized with PBS containing $0.5 \%$ Triton X-100. Next, the cells were blocked with the pre-hybridization solution for $4 \mathrm{~h}$ at $37^{\circ} \mathrm{C}$ and hybridized with PITPNA-AS1 specific probe (RiboBio) overnight at $37{ }^{\circ} \mathrm{C}$ followed by washing with the hybridization solution at $42{ }^{\circ} \mathrm{C}$ in the dark. Afterwards, 4'-6-diamidino2-phenylindole (DAPI) was utilized to stain nucleus for $10 \mathrm{~min}$, and a fluorescence microscope (Olympus) was used to capture the images of the cells.

\section{Cell counting kit-8 (CCK-8) assay}

Based on manufacturer's requirements, cell viability was testified by a CCK-8 kit (Boster Biological Technology, CA, USA). In brief, transfected HCC1937 and MDAMB-468 cells were plated into 96-well plates at a density of $2 \times 10^{3}$ cells/well and were incubated for 0,24 , 48 and $72 \mathrm{~h}$. Next, $10 \mu \mathrm{L}$ of CCK-8 solution was added into each well for further incubation in $5 \% \mathrm{CO}_{2}$ at $37{ }^{\circ} \mathrm{C}$ for $1 \mathrm{~h}$ after cell adhesion. The culture medium was then removed, and the plates were washed twice by PBS. Lastly, absorbance at $450 \mathrm{~nm}$ was monitored by a microplate reader (EL340; Bio-Tek Instruments, Hopkinton, MA, USA) for detecting cell viability.

\section{Colony formation assay}

Colony formation assay was performed to reveal cell proliferation. In brief, $1 \times 10^{3}$ HCC1937 and MDA-MB-468 cells were seeded in 6-well plates. At 2 weeks after incubation, colonies were fixed by methanol for $15 \mathrm{~min}$ and were dyed by crystal violet (Sigma-Aldrich) in PBS for $20 \mathrm{~min}$. After that, crystal violet was slowly washed away with running water. The plates were air-dried in an inverted position and number of stained colonies were manually counted. 


\section{Flow cytometry analysis}

Transfected HCC1937 and MDA-MB-468 cells were subjected to the staining of propidium iodide (PI) and FITCAnnexin V for detection of apoptosis rate. HCC1937 and MDA-MB-468 cells were seeded in 6-well plates at a concentration of $1 \times 10^{5}$ cells/well, after which cells were incubated in $5 \mu \mathrm{L}$ of Annexin V-FITC for $10 \mathrm{~min}$ and $10 \mu \mathrm{L}$ of PI for $15 \mathrm{~min}$ at $4{ }^{\circ} \mathrm{C}$ in the dark. Later, using CellQuest software (BD Biosciences, San Jose, CA), cells were analyzed by flow cytometer (FACScan; BD Biosciences), and apoptosis rate of TNBC cells was assessed. The early apoptotic cells were distributed in the second quadrant and late apoptotic and necrotic cells were in the third and fourth quadrants. Apoptosis rate (\%) was defined as cell percentage in the third quadrant.

\section{Wound healing assay}

Wound healing assay was performed to detect cell migration. TNBC cells $\left(1.5 \times 10^{6}\right)$ were plated into the 6-well plates. Then, a pipette tip was utilized to horizontally scratch the wound after cell reached about $80 \%$ confluence. After cells were washed twice, cells were incubated in serum-free medium for $24 \mathrm{~h}$. The gap between cells was photographed under a low-magnification phase-contrast microscope (Olympus MK, Tokyo, Japan).

\section{Transwell assay}

Transwell assay was conducted to reveal cell migration in vitro. Matrigel-coated upper chamber $(8.0 \mu \mathrm{m}$ pore size, BD Biosciences) was filled with TNBC cells $\left(5 \times 10^{4} /\right.$ well) suspended in DMEM. The DMEM supplemented by $10 \%$ FBS was placed in the bottom chamber. Fortyeight hours later, cells in the upper chamber were scraped off by cotton swabs. Cells in the lower chamber were fixed with methanol and stained with $0.5 \%$ crystal violet. Finally, the average number of stained cells were counted using an inverted microscope (Olympus) under five randomly selected visual fields by Image J software.

\section{Western blot analysis}

RIPA lysis buffer containing protease inhibitors was used for the extraction of total protein from HCC1937 and MDA-MB-468 cells. Before being transferred to a polyvinylidene fluoride membrane (Millipore), equivalent proteins were isolated by $10 \%$ sodium dodecyl sulfate polyacrylamide gel electrophoresis. After being blocked with $5 \%$ skimmed milk for $1 \mathrm{~h}$, the membranes were incubated with primary antibodies against E-cadherin (ab40772, 1/20000), N-cadherin (ab76011, 1/5000), Vimentin (ab92547, 1/200), Slug (ab27568, 1/500), Twist (ab175430, 1/1000), SIK2 (ab53423, 1/1000), DDX54 (ab76947, 1/2000), and GAPDH (ab9485, 1/2500) overnight at $4{ }^{\circ} \mathrm{C}$. Next, secondary antibody (ab6721, 1/10000) conjugated with HRP was added for further incubation of $1 \mathrm{~h}$ at room temperature. All the antibodies were purchased from Abcam (Cambridge, USA). A chemiluminescence detection system was applied to visualize the proteins. The gray value of each protein band was analyzed by the ImageJ software, and the ratio of the gray value of the target protein to GAPDH was calculated.

\section{In vivo experiment}

For animal study, a total of 20 female BALB/C nude mice (6-week-old; weighing 20-30 g) were purchased from Shi Laike Company. The animal experiments were performed with ethical approval from the Second Hospital of Shanxi Medical University (Shanxi, China). The nude mice were subcutaneously injected with $0.2 \mathrm{~mL}$ suspension of HCC1937 cells $\left(3 \times 10^{6}\right)$ that were stably transfected with sh-PITPNA-AS1 or sh-NC. The tumor volume was measured every 4 days. Volume $\left(\mathrm{mm}^{3}\right)=1 / 2 \mathrm{~L} \times \mathrm{D}^{2}$. $\mathrm{L}$ represents the maximum diameter, and $\mathrm{D}$ represents the shortest diameter of the tumor. After 28 days, mice were euthanized, and tumors were excised for weighing.

\section{Immunohistochemistry staining (IHC)}

Tumor tissues were fixed in $4 \%$ paraformaldehyde, and then dehydrated in ethanol solutions. Next, tissues were embedded in paraffin, and cut into $4 \mu \mathrm{m}$ sections. The sections were routinely deparaffinized, followed by antigen retrieval. After washing in PBS for 15 min, tissues were blocked with goat serum for $1 \mathrm{~h}$ followed by incubation with primary antibody against Ki67 (ab92742, 1/500) overnight at $4{ }^{\circ} \mathrm{C}$. Subsequently, the sections were cultivated with HRP-conjugated secondary antibody (ab6721, 1/1000). The slides were then counterstained with hematoxylin for $1 \mathrm{~min}$ and mounted with neutral balsam. The Ki67 positivity was detected using a Histostain ${ }^{\text {TM }}$ SP-9000 immunohistochemical staining kit (Zymed Laboratories, South San Francisco, CA, USA). An OLYMPUSBX-41 microscope (Olympus) was used to capture the images.

\section{RNA pull down assay}

HCC1937 and MDA-MB-468 cells were transfected with $50 \mathrm{nM}$ biotin-labeled PITPNA-AS1 for $48 \mathrm{~h}$. Cells were then incubated in RIPA lysis buffer for $10 \mathrm{~min}$ and then the mixture were centrifuged at $14,000 \times g$ to obtain the supernatant. Protein lysate was incubated with M-280 streptavidin beads which were pre-coated with RNasefree bovine serum albumin and yeast tRNA. Next, the beads were incubated at $4{ }^{\circ} \mathrm{C}$ for $3 \mathrm{~h}$. The binding miR520d-5p was purified by TRIzol and its expression was examined by RT-qPCR. As per manufacture's guidelines, a Pierce Magnetic RNA-Protein Pull-Down Kit 
(Thermo Fisher Scientific) was employed to detect the proteins binding with PITPNA-AS1. Protein extracts from HCC1937 or MDA-MB-468 cells were subjected to biotinylated PITPNA-AS1 (50 pmol) and M-280 streptavidin magnetic beads (Invitrogen) for $1 \mathrm{~h}$ at $4{ }^{\circ} \mathrm{C}$. Finally, the cells were washed five times with RIPA buffer, and incubated with $5 \times$ loading buffer at $95^{\circ} \mathrm{C}$ for $5 \mathrm{~min}$. The eluted DDX54, FMR1, IGF2BP1, IGF2BP2 proteins were detected by western blotting.

\section{Luciferase reporter assay}

For luciferase reporter assay, PITPNA-AS1-WT/Mut or SIK2-WT/Mut reporter was constructed by subcloning wild-type (WT) or mutant (Mut) sequences of PITPNAAS1 complementary to miR-520d-5p or SIK2 3'UTR into the pmirGLO dual-luciferase vector. Then, these constructed reporters were co-transfected with miR-520d-5p mimics or NC mimics into HCC1937 or MDA-MB-468 cells. For PITPNA-AS1 promoter-luciferase analysis, wild type or mutated PITPNA-AS1 promoter sequences (site 1 and site 2) were subcloned into the pGL3-Basis luciferase vector (Promega, Madison, WI, USA), and these constructs were co-transfected with pcDNA3.1/MYBL2 or pcDNA3.1 into HCC1937 and MDA-MB-468 cells. After 2 days, a Dual-Luciferase Report Assay System (Promega) was used for the measurement of luciferase activity. Relative luciferase activity was defined as the ratio of the relative light unit of firefly luciferase to that of Renilla luciferase.

\section{RNA immunoprecipitation (RIP) assay}

Based on the manufacturer's instruments, an EZ-Magna RIP kit (Millipore) was applied. In brief, HCC1937 and MDA-MB-468 cells were washed with pre-cooled PBS and then lysed in RIPA lysis buffer in an ice bath for $5 \mathrm{~min}$, and centrifuged at $12,000 \times \mathrm{g}$ for $10 \mathrm{~min}$ at $4{ }^{\circ} \mathrm{C}$. Then, cell extract was incubated with magnetic beads coated human anti-Ago2 (Millipore), anti-DDX54 (Millipore) or control anti-IgG (Millipore) at $4{ }^{\circ} \mathrm{C}$ overnight. Finally, expression of PITPNA-AS1, miR-520d-5p, SIK2 immunoprecipitated by Ago2 and expression of PITPNA-AS1, SIK2 immunoprecipitated by DDX52 were analyzed by RT-qPCR after being purified by proteinase $\mathrm{K}$.

\section{Actinomycin D assay}

Actinomycin D can inhibit the synthesis of mRNAs. After transfection with sh-NC, sh-PITPNA-AS1\#1, shDDX54\#1 for 48 h, HCC1937 and MDA-MB-468 cells were treated with actinomycin $\mathrm{D}(5 \mu \mathrm{mol} / \mathrm{L})$ for $0,4,8$, $12 \mathrm{~h}$. Later, RT-qPCR was used for the evaluation of relative SIK2 expression levels.
Chromatin immunoprecipitation (ChIP) assay

With a Magna ChIP Kit (Millipore), ChIP was performed to explore which site is responsible for the binding between MYBL2 and PITPNA-AS1 promoter. Briefly, crosslinked chromatin DNA was separated into fragments of 200-2000 bp through sonication. Next, lysates were immunoprecipitated with anti-MYBL2 or anti-IgG (internal control) at $4{ }^{\circ} \mathrm{C}$ overnight. Subsequently, the mixture was centrifuged, and the precipitate was washed with the low salt buffer, the high salt buffer, the $\mathrm{LiCl}$ solution, and the trace element solution. The protein-DNA complex was eluted with $250 \mu \mathrm{L}$ of ChIP Wash Buffer and de-crosslinked with $20 \mu \mathrm{L}$ of $5 \mathrm{M} \mathrm{NaCl}$. Quantity of immunoprecipitated DNA was detected by RT-qPCR.

\section{Statistical analysis}

Data were expressed as mean \pm standard deviation and were analyzed by SPSS 22.0 (SPSS, Chicago, USA) from three biological and technical replications. The variance significance was evaluated by Student's $t$ test for difference between two group or ANOVA for that among three groups. $\mathrm{P}<0.05$ was set as the threshold of statistical significance.

\section{Results \\ PITPNA-AS1 was upregulated in TNBC and localized in the cytoplasm}

First, the expression pattern of PITPNA-AS1 in TNBC was detected by RT-qPCR. PITPNA-AS1 expression was significantly upregulated in TNBC tissues compared to that in adjacent nontumor tissues (Fig. 1A). Moreover, PITPNA-AS1 expression in TNBC cell lines (HCC1937, MDA-MB-468, MDA-MB-231 and MDA-MB-436) showed a higher level compared to that in MCF10A cell line (Fig. 1B). HCC1937 and MDA-MB-468 cells were used for the following assays since they contained the relatively higher expression of PITPNA-AS1. The results of subcellular fractionation assay manifested that PITPNA-AS1 was majorly distributed in the cytoplasm of HCC1937 and MDA-MB-468 cells (Fig. 1C). Furthermore, results of FISH assay confirmed PITPNA-AS1 as a cytoplasmic RNA in TNBC (Fig. 1D), indicating that PITPNA-AS1 might regulate gene expression at the posttranscriptional level.

\section{PITPNA-AS1 knockdown suppressed TNBC cellular process} in vitro

Considering the upregulation of PITPNA-AS1 in TNBC, we then explored the functional role of PITPNA-AS1 by loss-of-function assays. PITPNA-AS1 expression was stably silenced in HCC1937 and MDA-MB-468 cells by transfection of sh-PITPNA-AS1\#1 and sh-PITPNAAS1\#2 (Fig. 2A). As showed in Fig. 2B, HCC1937 and 


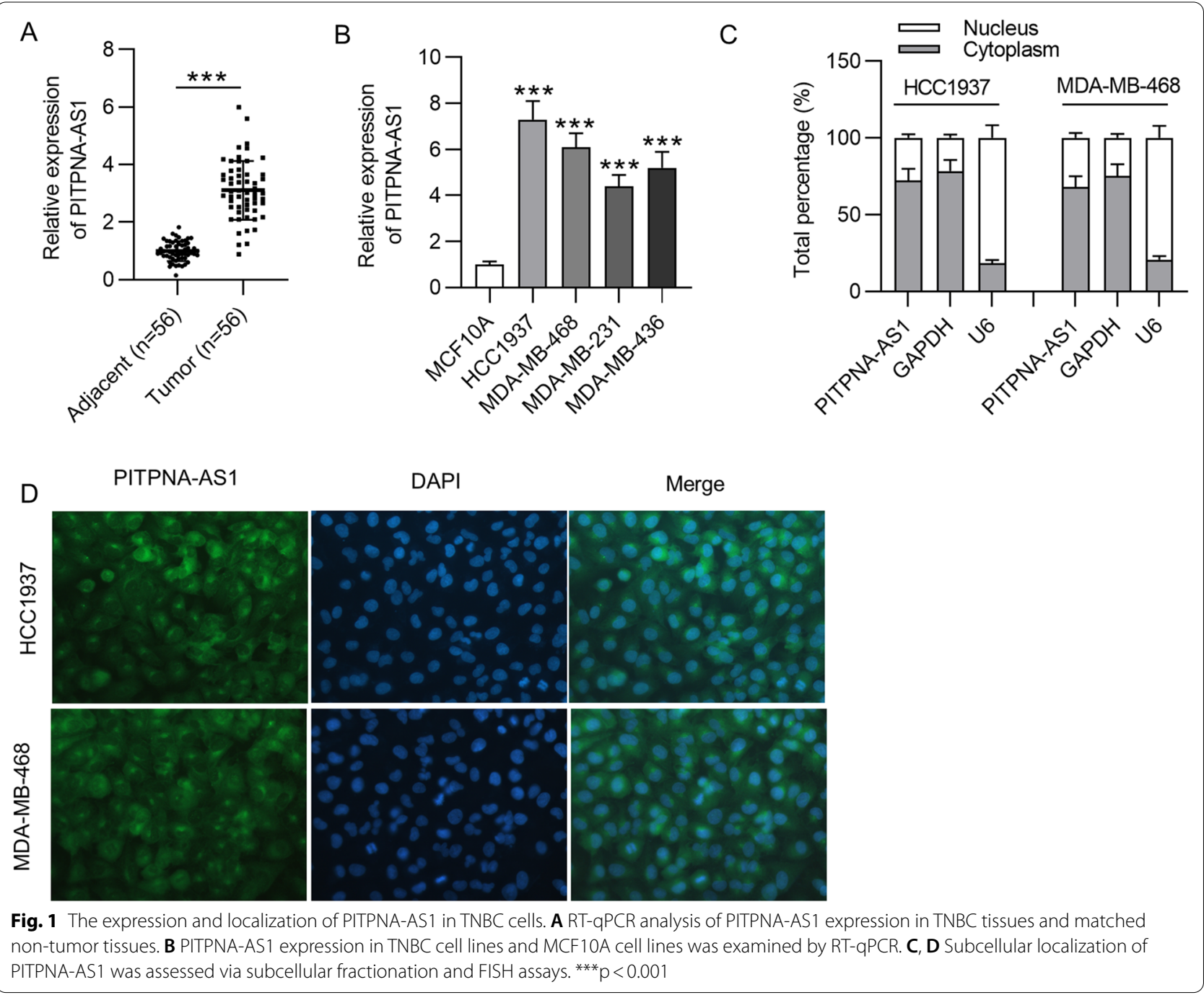

MDA-MB-468 cell viability was suppressed by silencing PITPNA-AS1. Moreover, results of colony formation assay demonstrated that PITPNA-AS1 suppressed HCC1937 and MDA-MB-468 cell proliferation (Fig. 2C). Furthermore, decreased PITPNA-AS1 expression promoted the apoptosis rate of HCC1937 and MDA-MB-468 cells (Fig. 2D). Through results of the wound healing assay, we found that the migrative ability of HCC1937 and MDA-MB-468 cells was inhibited by PITPNA-AS1 knockdown (Fig. 2E). Furthermore, downregulated PITPNA-AS1 suppressed the invasive capability of HCC1937 and MDA-MB-468 cells (Fig. 2F). Similarly, PITPNA-AS1 deficiency induced increase in E-cadherin protein level and decrease in N-cadherin, Vimentin, Slug and Twist protein levels, revealing that the epithelial-mesenchymal transition (EMT) process was inhibited by silenced PITPNA-AS1 in HCC1937 and MDA-MB-468 cells (Fig. 2G).
PITPNA-AS1 silencing inhibited tumor growth in vivo Later, the biological effect of PITPNA-AS1 on tumor growth in vivo was explored by animal experiments. At first, HCC1937 cells stably transfected with sh-PITPNAAS1\#1 or sh-NC were subcutaneously injected into nude mice. We found that tumor size in sh-PITPNA-AS1\#1 group was smaller than that in sh-NC group (Fig. 3A). Tumor growth in sh-PITPNA-AS1\#1 group was slower than that in sh-NC group (Fig. 3B). Furthermore, tumor volume and tumor weight were both reduced by PITPNA-AS1 knockdown (Fig. 3C, D). More importantly, results of IHC assay in xenograft tumors confirmed that PITPNA-AS1 depletion reduced expression of Ki67, suggesting that PITPNA-AS1 downregulation repressed cell proliferation in tumors (Fig. 3E). 

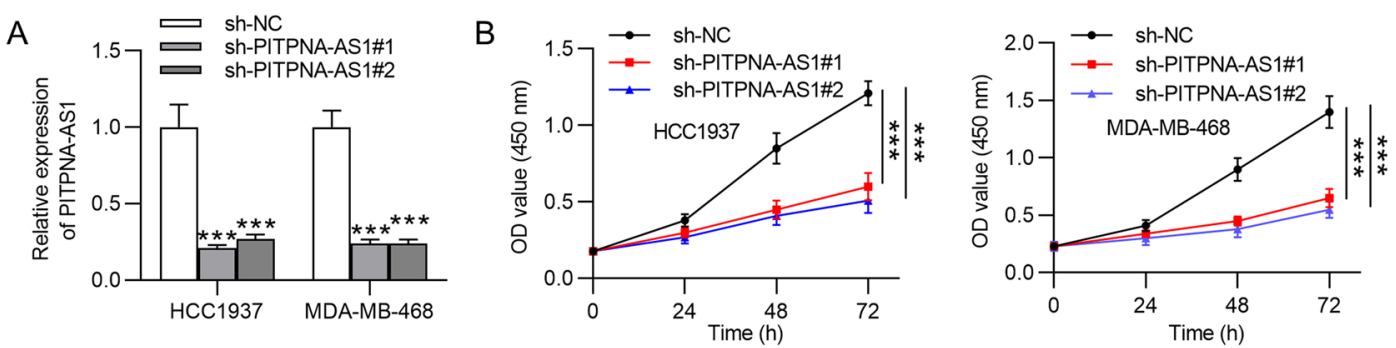

C
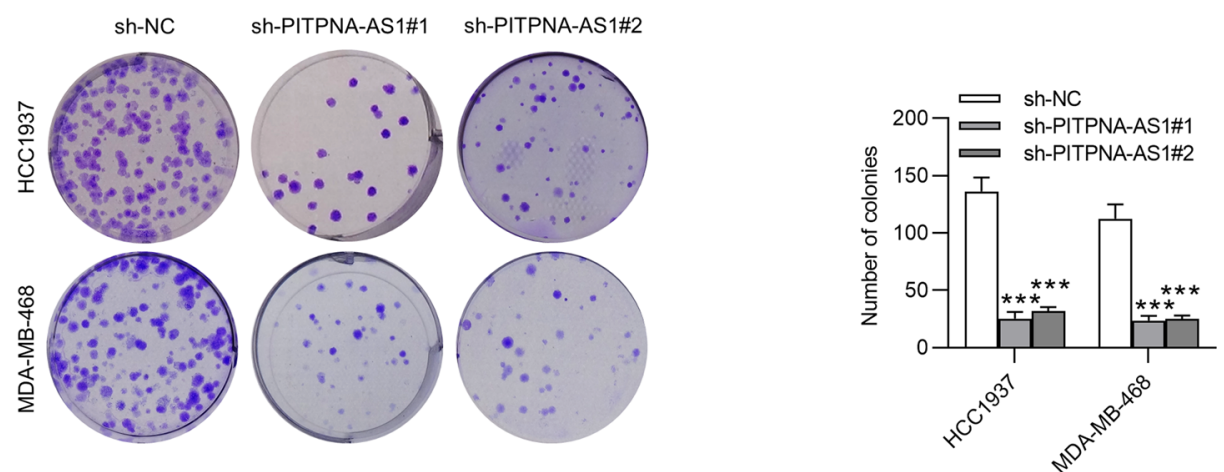

D
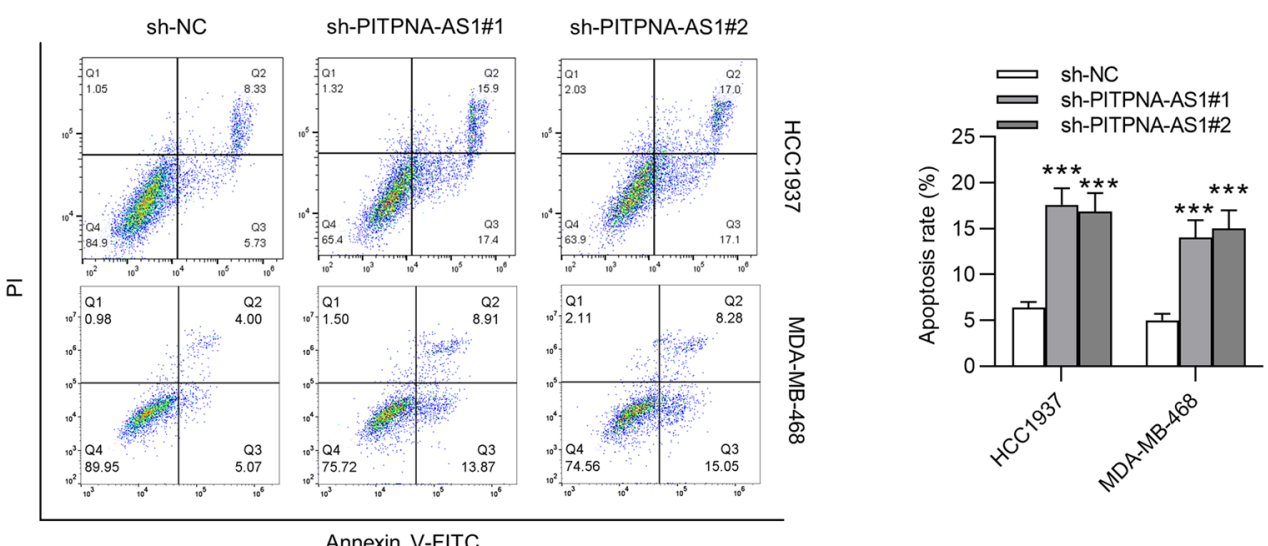

E
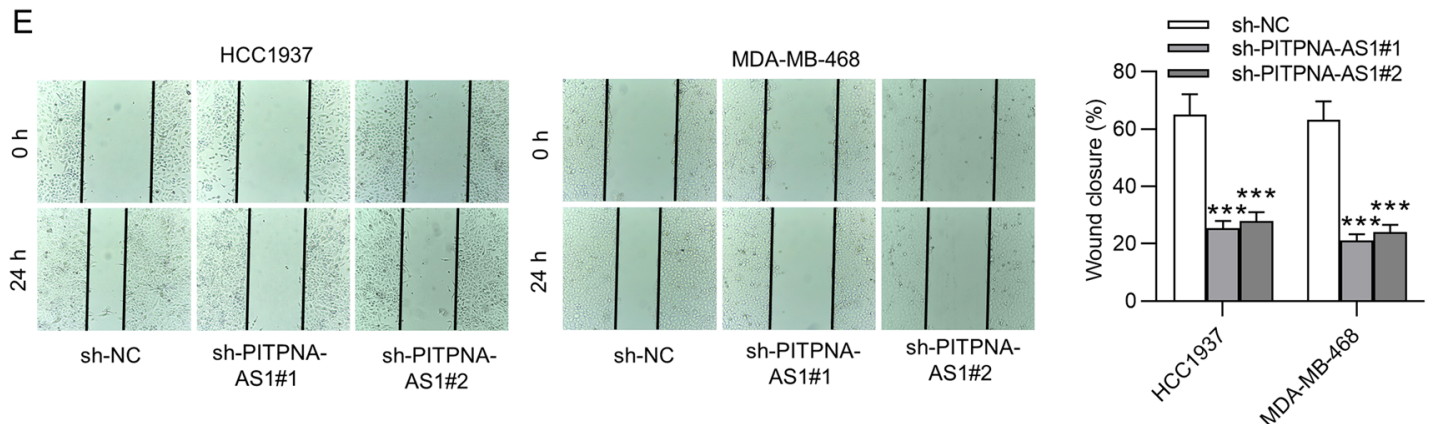

Fig. 2 The biological function of silenced PITPNA-AS1 in vitro. A RT-qPCR analysis was conducted to verify PITPNA-AS1 knockdown efficiency in HCC1937 and MDA-MB-468 cells. B, C CCK-8 and colony formation assays evaluated the proliferative ability of HCC1937 and MDA-MB-468 cells transfected with sh-PITPNA-AS1\#1/2 or sh-NC. D Flow cytometry analysis was conducted to detect the effect of PITPNA-AS1 knockdown on HCC1937 and MDA-MB-468 cell apoptosis. E, F Cell migration and invasion in PITPNA-AS1-silenced cells was analyzed by wound healing and Transwell assays. G Levels of proteins related to the EMT process were tested by western blot analysis in sh-PITPNA-AS1\#1/2 or sh-NC group. $* * * p<0.001$ 


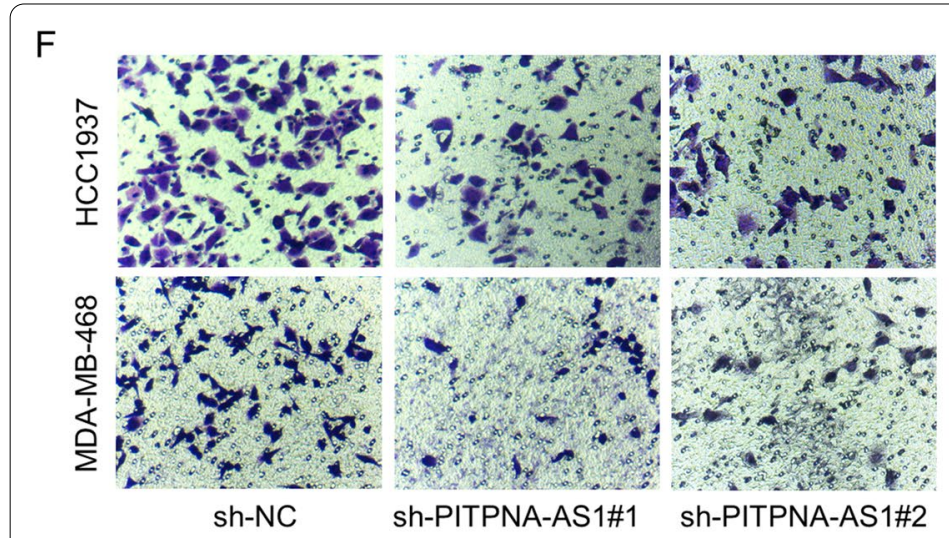

G

HCC1937
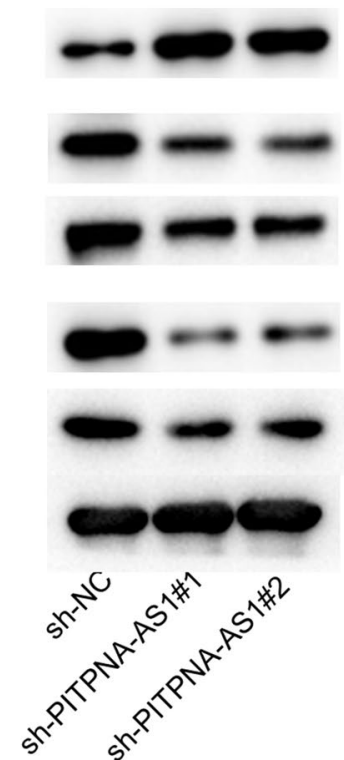

MDA-MB-468

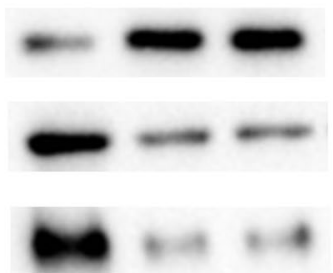

(54 kDa)

Slug

(30 kDa)

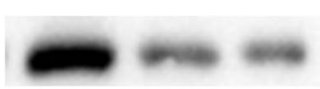

Twist

(21 kDa)

GAPDH

(36 kDa)

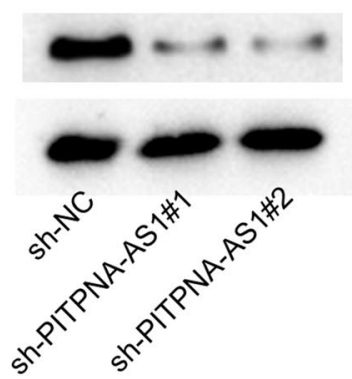

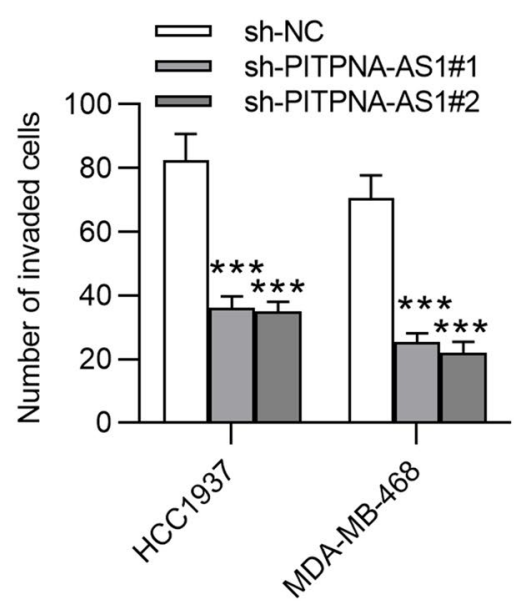

Fig. 2 continued

\section{PITPNA-AS1 sequestered miR-520d-5p in TNBC cells}

Based on the post-transcriptionally regulatory role of PITPNA-AS1 in TNBC cells, we speculated that PITPNA-AS1 might sponge specific miRNA to regulate TNBC progression through the ceRNA network. We searched the potential miRNAs for PITPNA-AS1 using starBase (http://starbase.sysu.edu.cn/). Under the condition of CLIP data $\geq 3$, Degradome Data $\geq 3$, two putative miRNAs (miR-524-5p and miR-520d-5p) were discovered (Fig. 4A). Further, RNA pull-down assay showed high enrichment of miR-520d-5p in PITPNA-AS1 biotin probe group, while miR-524-5p was not significantly enriched by the PITPNA-AS1 biotin probe (Fig. 4B), indicating the potential binding of PITPNA-AS1 to
miR-520d-5p but not miR-524-5p. Later, a remarkable downregulation of miR-520d-5p was detected in TNBC tissues by RT-qPCR (Fig. 4C). Furthermore, low expression level of miR-520d-5p was also observed in TNBC cell lines (Fig. 4D). Subsequently, miR-520d-5p mimics was transfected into HCC1937 and MDA-MB-468 cells to overexpress miR-520d-5p for further analysis (Fig. 4E). In addition, a complementary binding sequence between PITPNA-AS1 and miR-520d-5p was revealed by starBase (Fig. 4F). To confirm the interaction of PITPNA-AS1 and miR-520d-5p, luciferase reporter assay was conducted. The results revealed the weakened luciferase activity of PITPNA-AS1-WT induced by miR-520d-5p upregulation. No significant difference was found in luciferase 
A

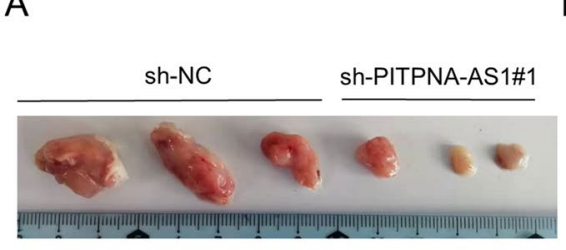

D

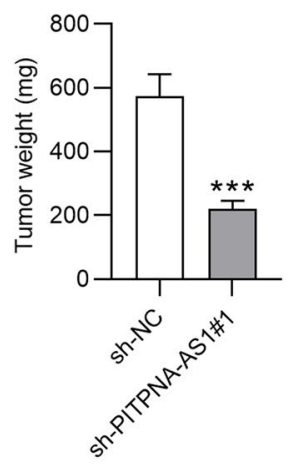

B

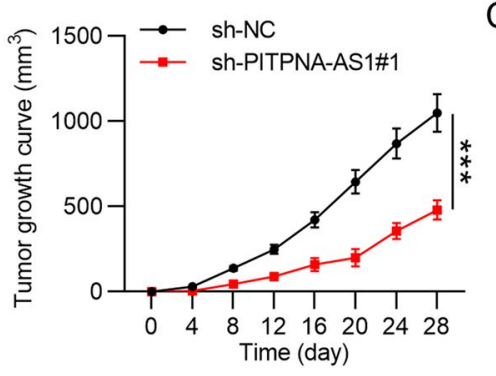

C

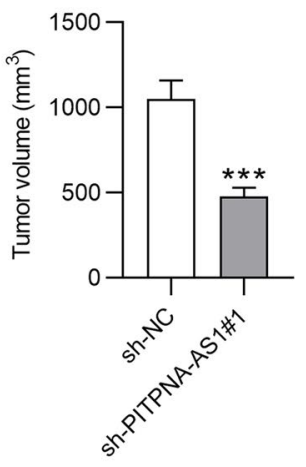

E

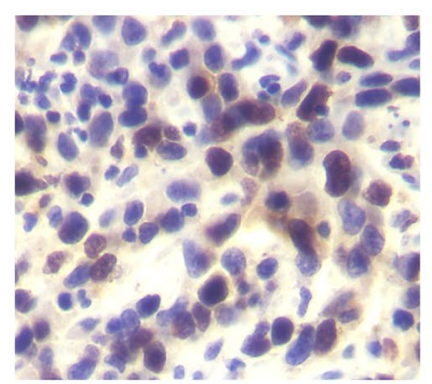

sh-NC
Ki67

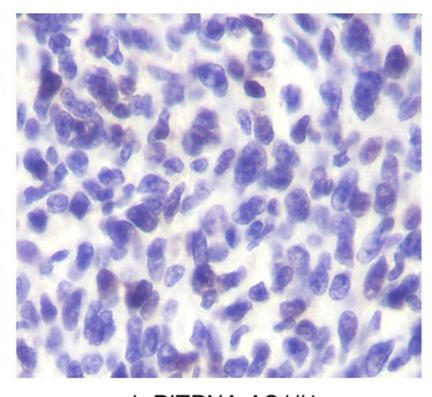

sh-PITPNA-AS1\#1

Fig. 3 Knockdown of PITPNA-AS1 suppressed TNBC tumor growth. A Tumors excised from the nude mice in sh-PITPNA-AS1\#1 and sh-NC group. B-D Tumor growth, volume and weight were analyzed after nude mice were subcutaneously injected with transfected HCC1937 cells. E Ki67 expression in tumors was shown by IHC assay after silencing PITPNA-AS1. ${ }^{* * *} \mathrm{p}<0.001$

activity of PITPNA-AS1-Mut in HCC1937 and MDAMB-468 cells after transfection of miR-520d-5p mimics compared to after transfection of NC mimics (Fig. 4G).

\section{SIK2 was the target gene of miR-520d-5p in TNBC cells}

To further support hypothesis of PITPNA-AS1 mediated ceRNA mechanism, miR-520d-5p target genes in TNBC were explored. Combining results from two online tools (RNA22 and microT), seven mRNAs were predicted as the downstream genes of miR-520d-5p (Fig. 5A). Expression of these mRNAs in TNBC tissues was testified by RT-qPCR analysis and only SIK2 was discovered to be prominently upregulated in TNBC tissues (Fig. 5B). Subsequently, SIK2 expression was higher in TNBC cell lines in comparison with that in MCF10A cell line (Fig. 5C). Afterwards, starBase predicted a binding site between miR-520d-5p and SIK2 (Fig. 5D). Luciferase reporter assay manifested that miR-520d-5p predominantly suppressed the luciferase activity of SIK2-WT but not that of SIK2-Mut (Fig. 5E). Moreover, PITPNA-AS1, miR-520d-5p and SIK2 were validated to co-exist in RNA-induced silencing complexes (RISCs) by RIP assay (Fig. 5F). Additionally,
miR-520d-5p expression was inhibited in HCC1937 and MDA-MB-468 cells with transfection of miR520d-5p inhibitor (Fig. 5G). We found that decreased mRNA and protein expression of SIK 2 by PITPNA-AS1 knockdown was partly reversed by miR-520d-5p downregulation, suggesting that, in addition to miR-520d-5p, PITPNA-AS1 can regulate SIK2 expression by other molecules (Fig. 5H).

\section{PITPNA-AS1 recruited DDX54 protein to stabilize SIK2 MRNA}

There was another post-transcriptional regulation of lncRNA maintaining mRNA stability by recruiting RBPs [19]. Based on above findings, we hypothesized that PITPNA-AS1 might modulate SIK2 expression not only by serving as a ceRNA but also by interacting with RBPs. SIK2 mRNA expression in TNBC cells treated with actinomycin D was detected by RT-qPCR. Results implied that PITPNA-AS1 knockdown inhibited SIK2 mRNA stability compared with sh-NC group (Fig. 6A). Subsequently, the potential RBPs that could interact with PITPNA-AS1 and SIK2 were explored. Through starBase, 4 RBPs (DDX54, 


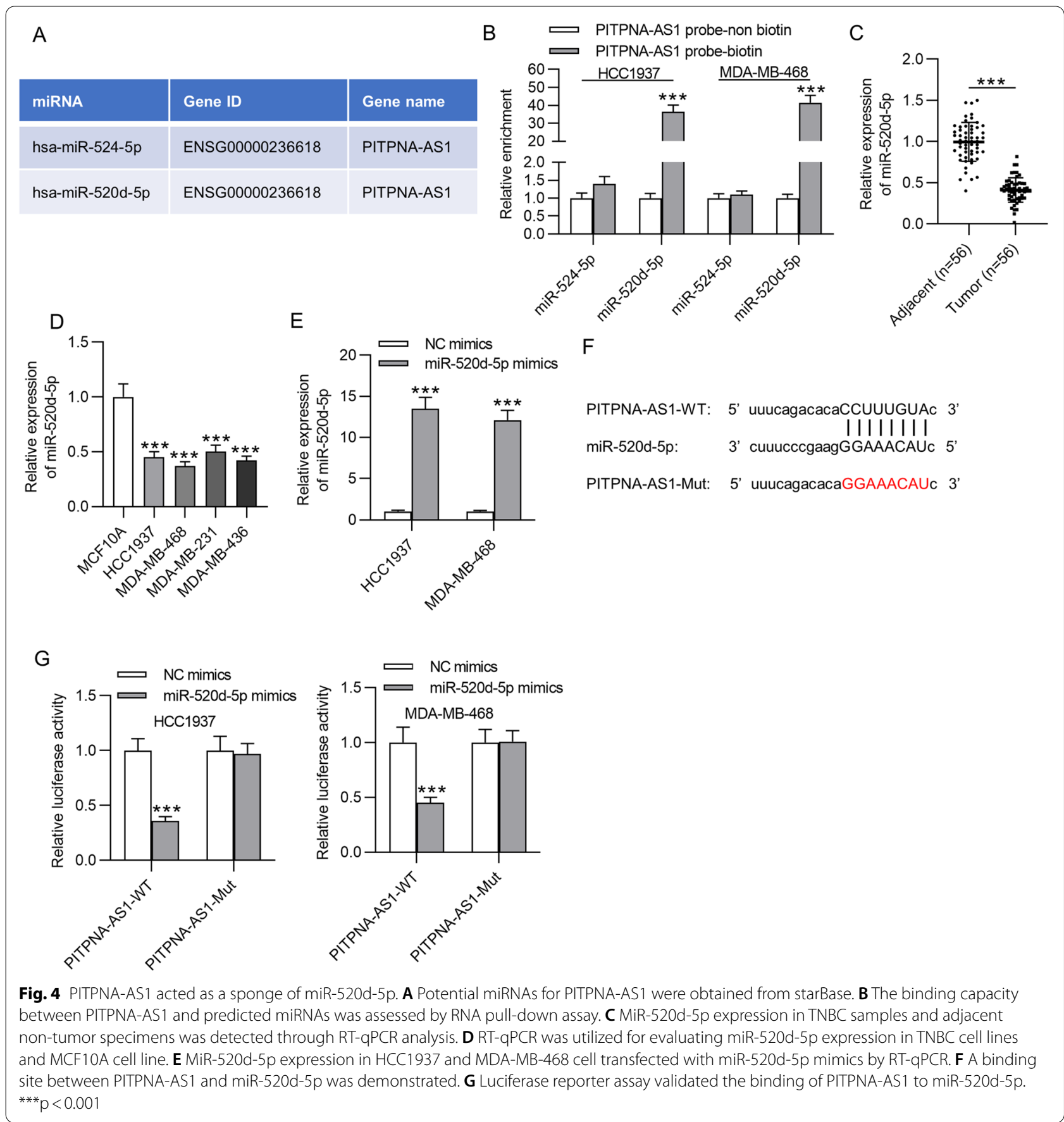

FMR1, IGF2BP1 and IGF2BP2) were found (Fig. 6B). To narrow the selection, RNA pull-down assay was conducted, and the results suggested that only DDX54 could bind to PITPNA-AS1 in HCC1937 and MDA-MB-468 cells (Fig. 6C). Subsequently, to testify whether DDX54 exerted function on SIK2 mRNA stability, we knocked down DDX54 in HCC1937 and MDA-MB-468 cells with transfection of sh-DDX54\#1/2, denoting sh-DDX54\#1 with better knockdown efficiency (Fig. 6D). SIK2 mRNA stability was significantly decreased by DDX54 downregulation in HCC1937 and MDA-MB-468 cells treated with actinomycin D (Fig. 6E). Later, results of RIP assay further confirmed that DDX54 protein combined with PITPNA-AS1 and SIK2 (Fig. 6F). In addition, PITPNAAS1 silencing weakened the interaction between DDX54 protein and SIK2 mRNA (Fig. 6G). Results of DDX54 


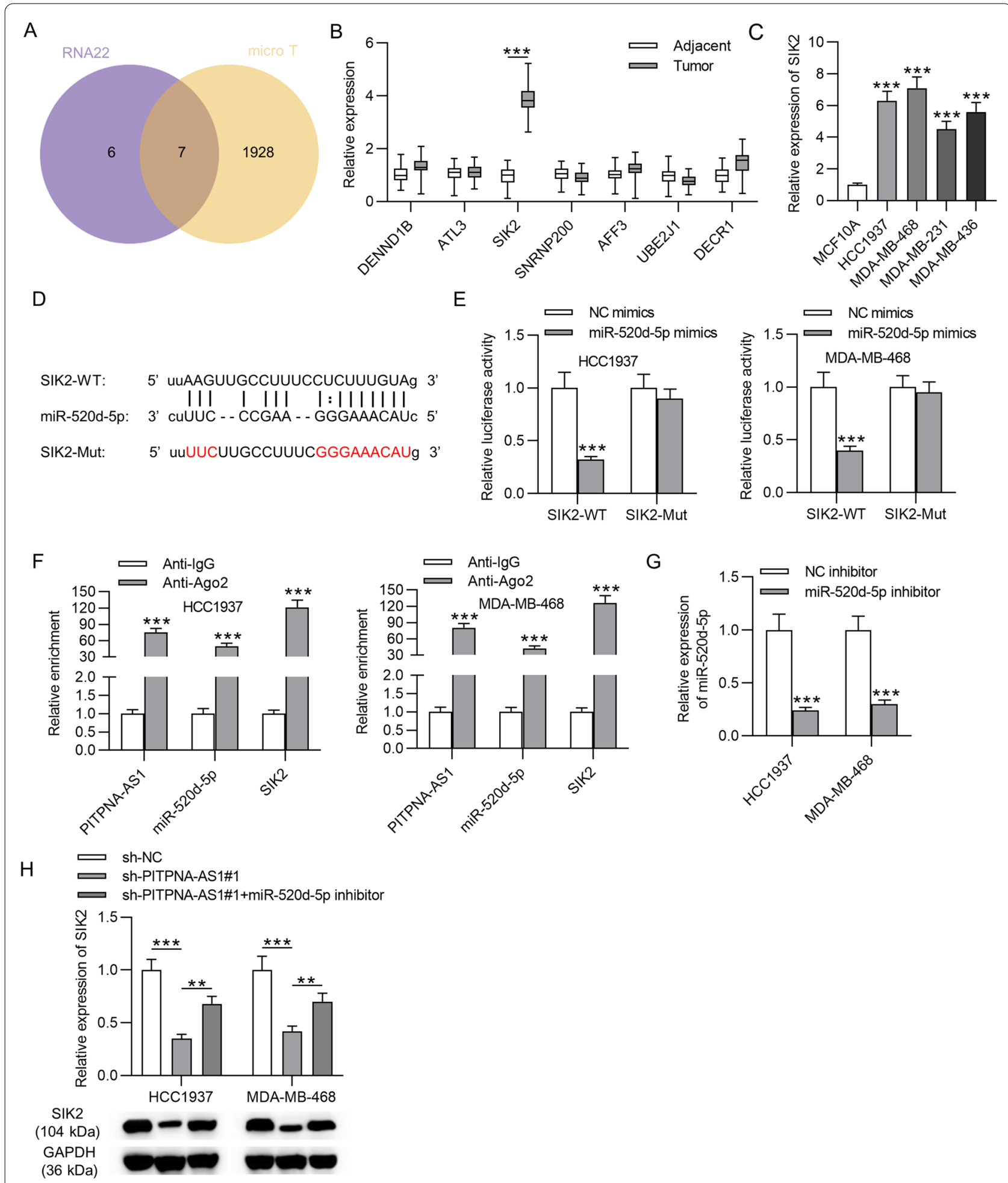

Fig. 5 MiR-520d-5p targeted SIK2 in TNBC. A Target mRNAs for miR-520d-5p based on prediction of DIANA and microT databases. B Expression levels of predicted mRNAs in TNBC tissues and adjacent non-tumor tissues were determined by RT-qPCR. C SIK2 expression in TNBC cells and MCF10A cell line was detected via RT-qPCR. D The binding sequence of miR-520d-5p on SIK2 3'UTR was predicted through starBase. E The binding of miR-520d-5p to SIK2 was confirmed by luciferase reporter assay. F RIP assay showed the enrichments of PITPNA-AS1, miR-520d-5p and SIK2 in the beads-conjugated with anti-lgG or anti-Ago2. G The efficiency of miR-520d-5p inhibition in HCC1937 and MDA-MB-468 cells was examined by RT-qPCR. H SIK2 expression in HCC1937 and MDA-MB-468 cells in each group was detected by RT-qPCR and western blotting analyses. ${ }^{* *} p<0.01$, ${ }^{* * *} p<0.001$ 


\section{(See figure on next page.)}

Fig. 6 PITPNA-AS1 increased SIK2 mRNA stability via recruiting DDX54. A SIK2 mRNA stability in HCC1937 and MDA-MB-468 cells transfected sh-PITPNA-AS1\#1 or sh-NC after treating with Actinomycin D was detected by RT-qPCR. B Four common RBPs for PITPNA-AS1 (CLIP Data $\geq 2$ ) and SIK2 (CLIP Data $\geq 5$ ) were predicted by starBase. C The binding capacity of PITPNA-AS1 to DDX54, FMR1, IGF2BP1 or IGF2BP2 was detected by RNA pull down assay. D Efficiency of silencing of DDX54 in HCC1937 and MDA-MB-468 cells was identified via RT-qPCR and western blot. E Stability of SIK2 mRNA in transfected cells treated with actinomycin D for $0,4,8,12 \mathrm{~h}$ was evaluated by RT-qPCR. $\mathbf{F}$ The interaction of DDX54 protein with PITPNA-AS1 or SIK2 mRNA was verified through RIP assay. G RIP assay demonstrated the changes in the binding of DDX54 protein to SIK2 mRNA upon PITPNA-AS1 knockdown. H DDX54 expression at the mRNA and protein levels after silencing PITPNA-AS1 was measured by RT-qPCR and western blot. I RT-qPCR and western blot verified the overexpression efficiency of DDX54. J RT-qPCR and western blot analyses of SIK2 expression in HCC1937 and MDA-MB-468 cells transfected with indicated plasmids. ${ }^{* *} p<0.01,{ }^{* * *} p<0.001$

mRNA and protein levels remained unchanged in shPITPNA-AS1\#1 transfected TNBC cells, suggesting that PITPNA-AS1 recruited DDX54 to maintain SIK2 mRNA rather than regulating DDX54 expression (Fig. 6H). DDX54 overexpression efficiency was confirmed by RTqPCR and western blotting (Fig. 6I). As shown in Fig. 6J, inhibited SIK2 mRNA and protein expression by silenced PITPNA-AS1 was partly countervailed by overexpressed DDX54, whereas was fully recovered by DDX54 upregulation and miR-520d-5p inhibition.

\section{PITPNA-AS1 upregulated SIK2 to play an oncogenic role in TNBC cells by miR-520d-5p and DDX54}

Finally, rescue assays were conducted to further verify that mechanisms of PITPNA-AS1 mentioned above could drive the development of TNBC. Accordingly, SIK2 expression was upregulated in HCC1937 cells by transfecting pcDNA3.1/SIK2 (Fig. 7A). Through results of CCK-8 and colony formation assays, we found that cell viability and proliferation were inhibited by PITPNA-AS1 deficiency and was promoted by transfection of pcDNA3.1-SIK2 or cotransfection of miR-520d-5p inhibitor + pcDNA3.1-DDX54. The suppressive effects of PITPNA-AS1 deficiency onHCC1937 cell viability and proliferation were rescued by SIK2 overexpression or by upregulated DDX54 after inhibiting miR-520d-5p (Fig. 7B, C). Transfection of pcDNA3.1-SIK2 or cotransfection of miR-520d-5p inhibitor + pcDNA3.1-DDX54 inhibited apoptosis rate of HCC1937 cells. Cell apoptosis induced by silenced PITPNA-AS1 was inhibited by upregulated SIK2; this effect was also reversed by coeffect of suppressed miR-520d-5p and overexpressed DDX54 (Fig. 7D). In addition, cell migration and invasion inhibited by PITPNA-AS1 knockdown were offset by overexpressing SIK2 or by inhibiting miR-520d-5p expression together with upregulating DDX54 (Fig. 7E, F). Moreover, either SIK2 overexpression or miR-520d-5p inhibition together with DDX54 upregulation rescued the effect of silenced PITPNA-AS1 on the EMT process (Fig. 7G).

\section{MYBL2 transcriptionally activated PITPNA-AS1 expression in TNBC}

Transcription activation is a major cause for the aberrant expression of genes. Considering this, we explored whether upregulation of PITPNA-AS1 is attributed to this manner. Through UCSC (http://genome.ucsc.edu/), some potential transcription factors that could bind to PITPNA-AS1 promoter were found. Among which, E2F6, PAX5, FOXP2, ELK5, MYBL2, YY1 were found at a higher level in TNBC cells than in MCF10A cell line, as revealed by $\mathrm{RT}$-qPCR analysis (Additional file 1: Figure $\mathrm{S} 1 \mathrm{~A}$ ). Later, above-mentioned transcription factors were upregulated in HCC1937 and MDA-MB-468 cells by transfection of pcDNA3.1 overexpression plasmid for further analysis (Additional file 1: Figure S1B). Observed from Additional file 1: Figure S1C, we found that PITPNA-AS1 expression was predominantly increased in MYBL2-overexpressed cells. Further, we knocked down MYBL2, and discovered that MYBL2 silencing caused downregulation in PITPNA-AS1 expression level (Additional file 1: Figure S1D). Therefore, we hypothesized that PITPNA-AS1 could be transcriptionally activated by MYBL2 in TNBC cells. MYBL2 DNA motif and four putative binding sites of MYBL2 on PITPNA-AS1 promoter were predicted based on Jaspar online database (Additional file 1: Figure S1E). ChIP assay suggested the binding of MYBL2 to PITPNA-AS1 promoter in P1 section which contained site 1 and site 2 (Additional file 1: Figure S1F). Next, luciferase reporter assay was performed to further confirm the interaction of MYBL2 and PITPNA-AS1 promoter. We discovered that the luciferase activity of vectors containing WT and Mut 1 sequences of PITPNA-AS1 promoter was strengthened while that of vectors containing Mut 2 and Mut $1 / 2$ sequences of PITPNA-AS1 promoter remained unchanged after overexpressing MYBL2, indicating that MYBL2 interacted with PITPNA-AS1 promoter at site 2 (Additional file 1: Figure S1G). 

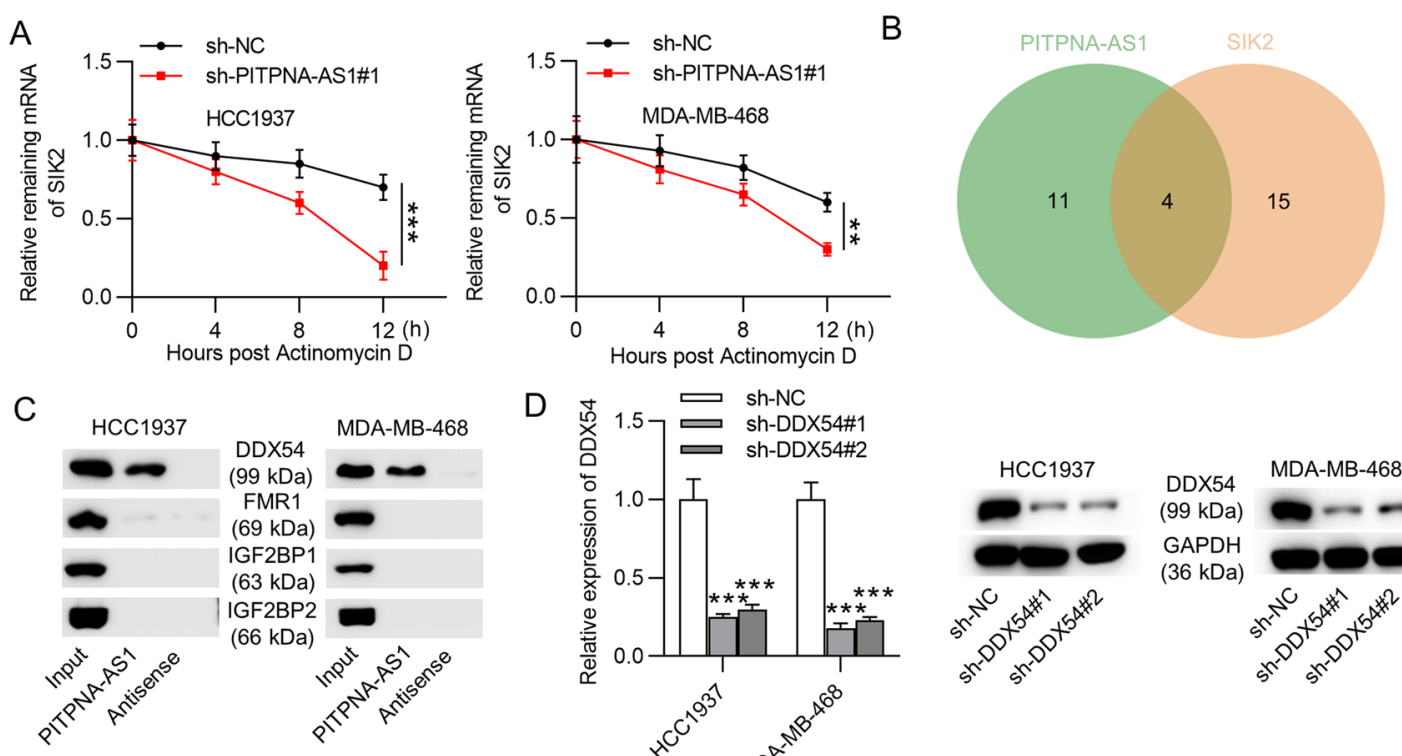

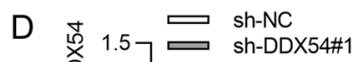
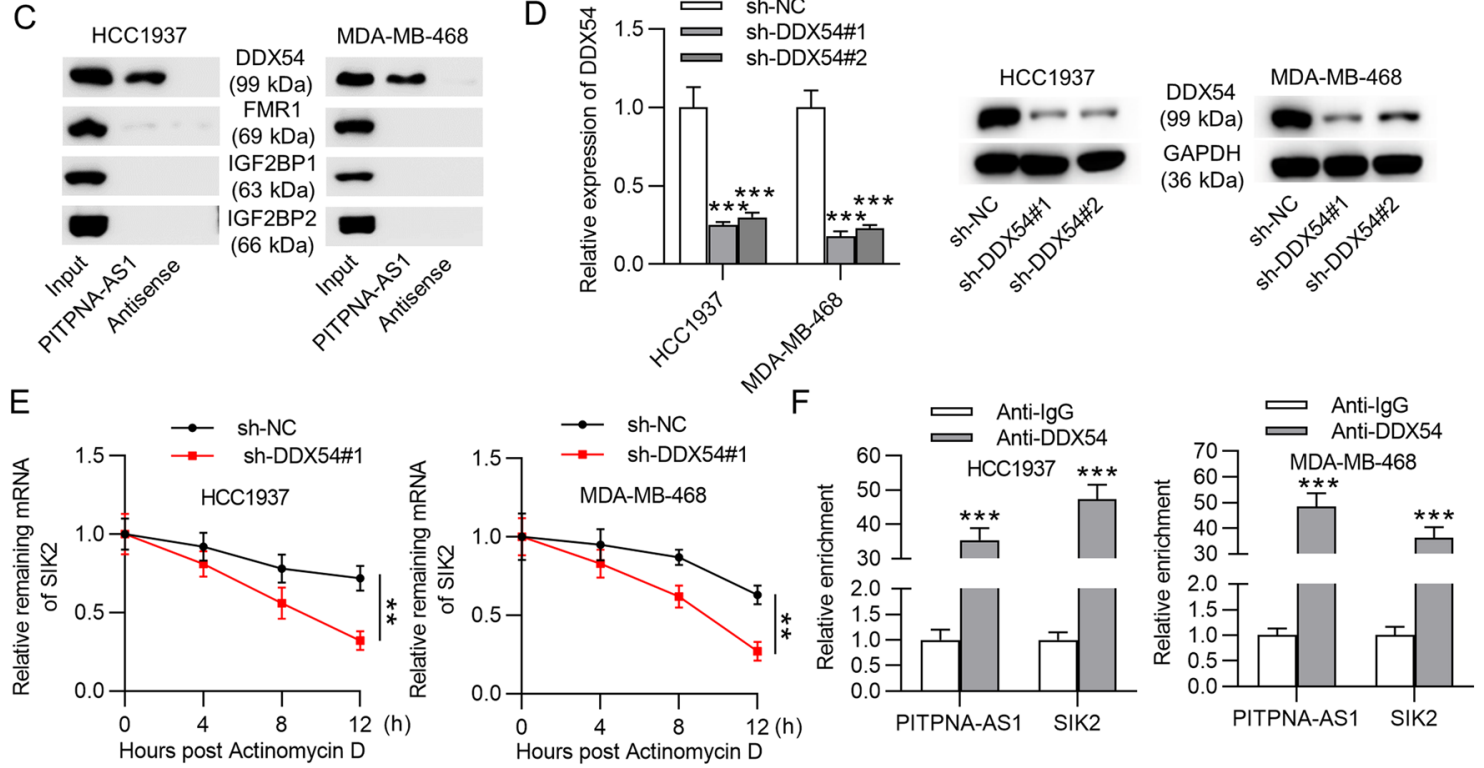

G
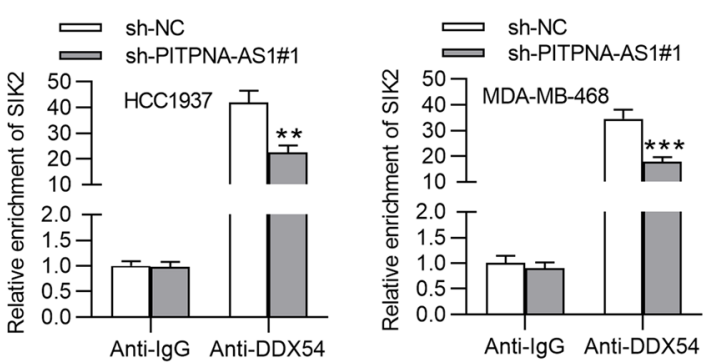

$\mathrm{H}$

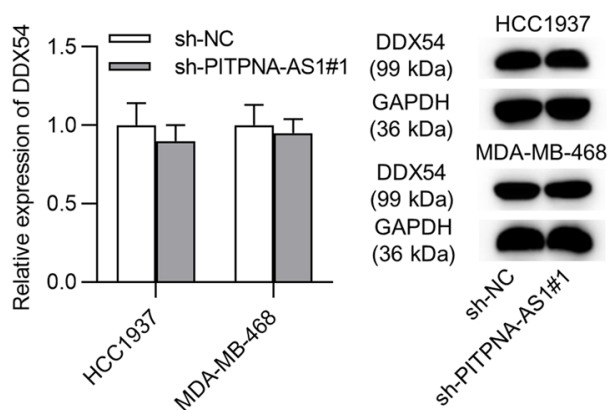

I

$\mathrm{J}$ a sh-NC

$\mathrm{b}$ sh-PITPNA-AS1\#1

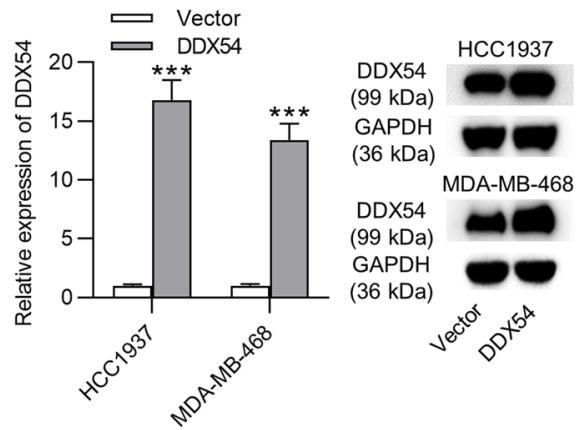

c sh-PITPNA-AS1\#1+DDX54

$\mathrm{d}=$ sh-PITPNA-AS1\#1+DDX54+miR-520d-5p inhibitor

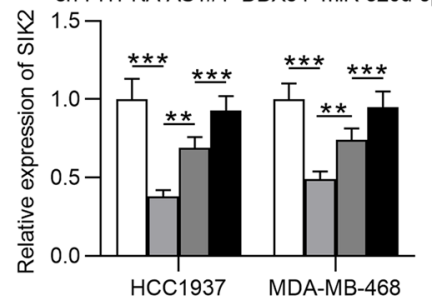

SIK2

HCC1937

(104 kDa)

GAPDH

(36 kDa)

SIK2

(104 kDa)

GAPDH

(36 kDa)

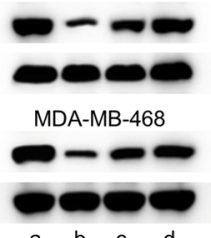

Fig. 6 (See legend on previous page.) 
A

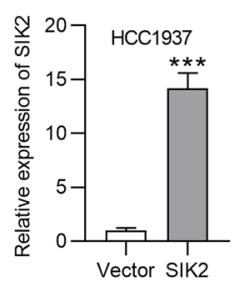

C
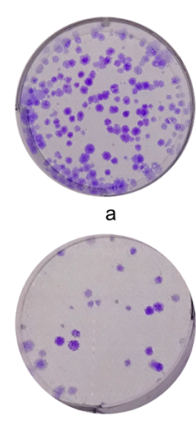

d

D

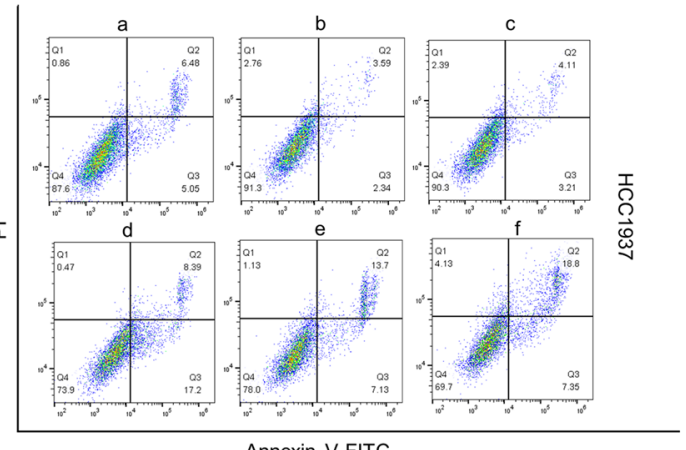

$E$
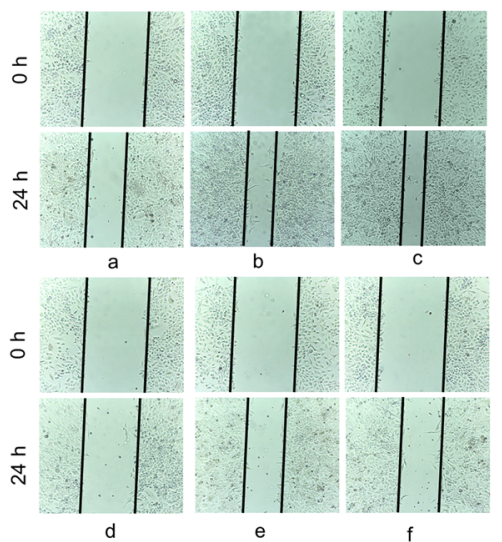

B $\quad$ a $\rightarrow$ sh-NC

$\mathrm{b} \rightarrow-$ sh-NC+SIK2

$c \rightarrow$ sh-NC+miR-520d-5p inhibitor+DDX54

$d \rightarrow$ Sh-PITPNA-AS1\#1

e $\rightarrow$ sh-PITPNA-AS1\#1+SIK2

$f \rightarrow$ sh-PITPNA-AS1\#1+miR-520d-5p inhibitor+DDX54

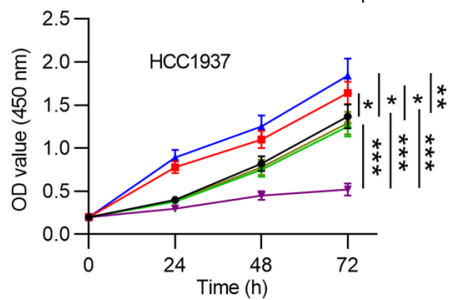

$\begin{array}{ll}a \rightleftharpoons & \text { sh-NC } \\ b & \text { sh-NC+SIK2 }\end{array}$

$c=$ sh-NC+miR-520d-5p inhibitor+DDX54

$\mathrm{d}=$ sh-PITPNA-AS1\#1

ए sh-PITPNA-AS1\#1+SIK2

f sh-PITPNA-AS1\#1+miR-520d-5p inhibitor+DDX54

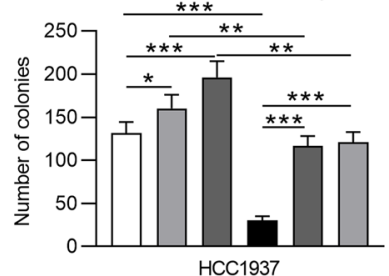

HCC1937

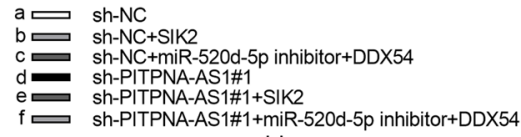

$f=$ sh-PITPNA-AS1\#1+miR-520d-5p inhibitor+DDX54

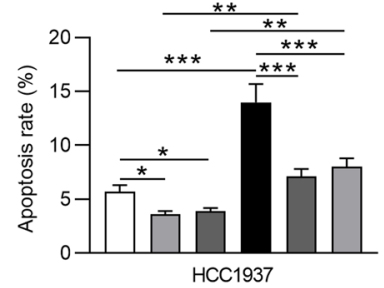

a sh-NC

b 5 Sh-NC+SIK2

d Sh-PITPNA-AS1\#1

e sh-PITPNA-AS1\#1+SIK2

$f \rightleftharpoons$ sh-PITPNA-AS1\#1+miR-520d-5p inhibitor+DDX54

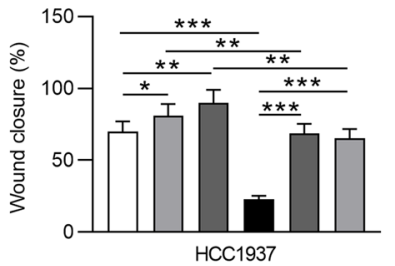

Fig. 7 PITPNA-AS1 promoted TNBC cellular processes by targeting the miR-520d-5p/DDX54/SIK2 axis. A SIK2 expression in HCC1937 cell lines transfected with pcDNA3.1 (Vector) or pcDNA3.1/SIK2 (SIK2) was examined via RT-qPCR. B, C The viability and proliferation of transfected cells were determined with CCK-8 and colony formation assays. D Flow cytometry analysis showed the apoptosis in each group. E, F Migration and invasion of cells with indicated transfections were measured by wound healing assay and Transwell assays. G EMT-associated proteins in the HCC1937 cell lines of indicated groups were tested by western blot. ${ }^{*} p<0.05,{ }^{* *} p<0.01,{ }^{* * *} p<0.001$ 
$\mathrm{F}$

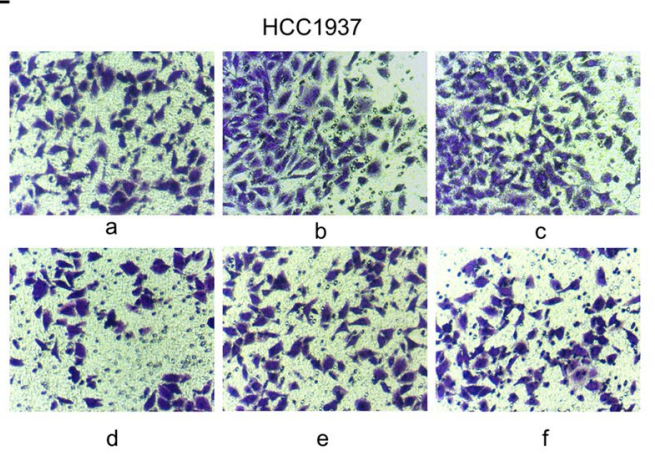

G

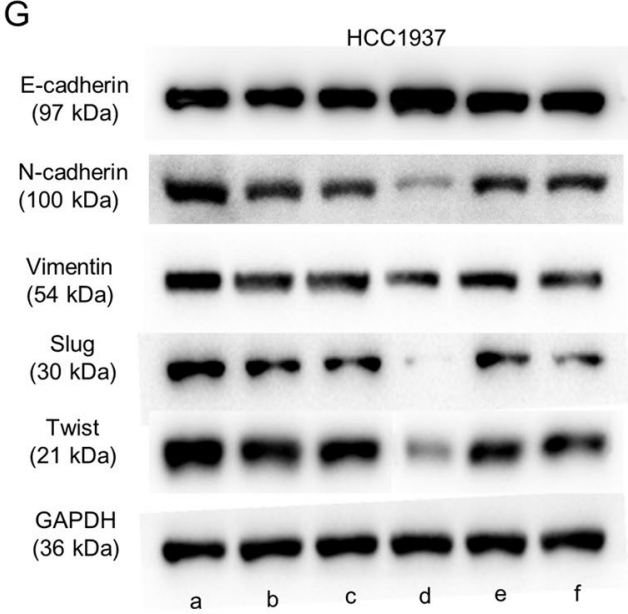

a sh-NC

$\mathrm{b} \rightleftharpoons \mathrm{sh}-\mathrm{NC}+\mathrm{SIK} 2$

$\mathrm{c} \rightleftharpoons$ sh-NC+miR-520d-5p inhibitor+DDX54

d Sh-PITPNA-AS1\#1

e sh-PITPNA-AS1\#1+SIK2

$\mathrm{f}$ sh-PITPNA-AS1\#1+miR-520d-5p inhibitor+DDX54

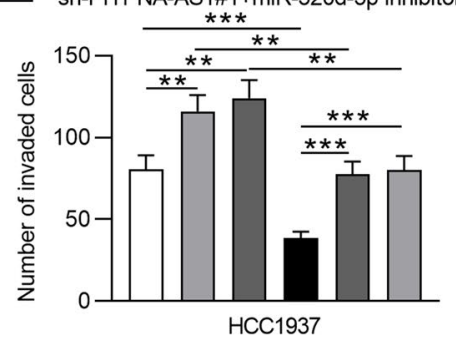

Fig. 7 continued

\section{Discussion}

Increasing literatures reported that lncRNAs are involved in occurrence and development of various human cancers by sponging miRNA or recruiting RBPs [23, 24]. Although the essential roles of abnormally expressed lncRNAs have been highlighted in TNBC progression $[14,25]$, the potential role and mechanism of IncRNA PITPNA-AS1 underlying TNBC remain obscure and deserve to be explored. Our study suggested that PITPNA-AS1 expression was higher in TNBC tissues and cells than in control tissues and cells, and mainly distributed in the cytoplasm of TNBC cells. Moreover, decreased PITPNA-AS1 repressed TNBC cell proliferation, facilitated cell apoptosis, and suppressed cell migration and invasion in vitro. Furthermore, PITPNA-AS1 silencing inhibited xenograft tumor growth in vivo. Collectively, PITPNA-AS1 exhibited oncogenic properties in TNBC.

MiRNAs are another class of ncRNAs with about 22-24 nucleotides in length and played important roles in cancer progression [26, 27]. Existing evidence has depicted that lncRNAs can combine with specific miRNA to facilitate or suppress the initiation or progression of tumors [28, 29]. In this study, miR-520d-5p was identified for further exploration via bioinformatics analysis and a series of molecular mechanism experiments. Previously, miR-520d-5p was found to inhibit cell proliferation and cell cycle via targeting PTTG1 in glioma [30]. In gastric cancer, miR-520d-5p is an important regulator in cell proliferation and survival [31]. In addition, miR-520d-5p functions as an anti-oncogene in colorectal cancer and suppresses tumor growth and metastasis via regulating CTHRC1 [32]. Herein, we found that miR-520d-5p had binding capacity with PITPNA-AS1 in TNBC. Furthermore, miR-520d-5p expression was at a low level in TNBC tissues and cell lines. These findings suggested that PITPNA-AS1 sequestered miR-520d-5p in TNBC.

Salt inducible kinase 2 (SIK2) has been validated to exert tumor-promoting functions in a variety of cancers, including TNBC $[33,34]$. Nevertheless, the relationship between SIK2 and miR-520d-5p (or PITPNA-AS1) in TNBC cells needs investigation. In our study, it was verified that SIK2 was directly targeted by miR-520d-5p in 
TNBC. More importantly, results in our study indicated that PITPNA-AS1 positively modulated SIK2 expression not merely via sponging miR-520d-5p.

DEAD-box helicase 54 (DDX54) was recognized as a member of RBPs and reported as an oncogene in some cancers $[35,36]$. In this study, SIK2 mRNA stability was inhibited by PITPNA-AS1 knockdown. DDX54 was identified to interact with PITPNA-AS1 (or SIK2) in TNBC cells. In addition, restoration experiments suggested that TNBC cellular processes inhibited by silenced PITPNAAS1 was rescued by SIK2 overexpression or co-effect of miR-520d-5p inhibition and DDX54 upregulation.

Emerging investigations have implied that transcriptional regulation mediated by transcription factor was a major reason for the aberrant expression of lncRNAs [37, 38]. MYB proto-oncogene Like 2 (MYBL2) was known as a transcription factor in lung adenocarcinoma [39]. Our study revealed that MYBL2 positively regulated PITPNAAS1 expression and bound to PITPNA-AS1 promoter, which indicated that the upregulation of PITPNA-AS1 in TNBC was transcriptionally induced by MYBL2.

\section{Conclusions}

Conclusively, our study showed that MYBL2-induced PITPNA-AS1 upregulated SIK2 expression to drive TNBC cellular processes via miR-520d-5p and DDX54. This might provide a meaningful theoretic basis for further exploration on TNBC therapy strategies.

\footnotetext{
Abbreviations

IncRNAs: Long non-coding RNAs; PITPNA-AS1: LncRNA PITPNA antisense RNA 1; BC: Breast cancer; ER: Estrogen receptor; PR: Progesterone receptor; TNBC: Triple-negative breast cancer; ncRNAs: Non-coding RNAs; ceRNA: Competing endogenous RNA; miRNA: MicroRNA; RBPs: RNA-binding proteins.
}

\section{Supplementary Information}

The online version contains supplementary material available at https://doi. org/10.1186/s12967-021-02956-6.

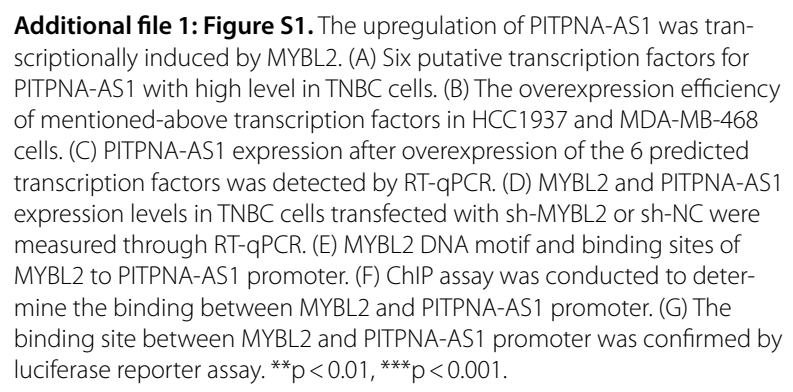
scriptionally induced by MYBL2. (A) Six putative transcription factors for PITPNA-AS1 with high level in TNBC cells. (B) The overexpression efficiency of mentioned-above transcription factors in HCC1937 and MDA-MB-468 cells. (C) PITPNA-AS1 expression after overexpression of the 6 predicted transcription factors was detected by RT-qPCR. (D) MYBL2 and PITPNA-AS1 expression levels in TNBC cells transfected with sh-MYBL2 or sh-NC were measured through RT-qPCR. (E) MYBL2 DNA motif and binding sites of MYBL2 to PITPNA-AS1 promoter. (F) ChIP assay was conducted to determine the binding between MYBL2 and PITPNA-AS1 promoter. (G) The binding site between MYBL2 and PITPNA-AS1 promoter was confirmed by luciferase reporter assay. ${ }^{* *} p<0.01,{ }^{* * *} p<0.001$.

\section{Authors' contributions}

FX and JJG participated in the literature search, analysis and interpretation of the data, and wrote the manuscript. BLL, PBY, LHW, and YY provided project guidance, revised the manuscript, and provision of experimental sites and funding. All authors read and approved the final manuscript.

\section{Funding}

This work was supported by Research Project Supported by Shanxi Scholarship Council of China (No. 2020-188), 2018 Hunan Science and Health Joint Fund (No. 2018JJ6122), and China International Medial Foundation (No. Z-2017-26-1902-2).

\section{Availability of data and materials}

All data from this study are available in this published article.

\section{Declarations}

Ethics approval and consent to participate

Written informed consent was obtained from all participants. The study complied with the Declaration of Helsinki and was approved by the Ethics Committee of The Second Hospital of Shanxi Medical University (Shanxi, China).

\section{Consent for publication}

Not applicable.

\section{Competing interests}

The authors declare that they have no competing interests.

\section{Author details}

${ }^{1}$ Department of Andrology, The First Affiliated Hospital of University of South China, Hengyang 421001, Hunan, China. ${ }^{2}$ Changsha Social Work College, Changsha 421004, Hunan, China. ${ }^{3}$ Department of Oncology, The Second Hospital of Shanxi Medical University, Taiyuan 030001, Shanxi, China. ${ }^{4}$ Department of Endocrinology and Metabolism, The Second Hospital of Shanxi Medical University, Taiyuan 030001, Shanxi, China. ${ }^{5}$ Department of Intensive Care Unit, Affiliated Nanhua Hospital, University of South China, No. 336, Dongfeng South Road, Zhuhui District, Hengyang 421001, Hunan, China. ${ }^{6}$ Department of General Surgery, The Second Hospital, University of South China, 30 Jiefang Road, Shigu District, Hengyang 421001, Hunan, China.

Received: 19 April 2021 Accepted: 20 June 2021

Published online: 05 August 2021

\section{References}

1. Cardoso F, Harbeck N, Barrios CH, Bergh J, Cortés J, El Saghir N, et al. Research needs in breast cancer. Ann Oncol. 2017;28(2):208-17.

2. Zhai Q, Li H, Sun L, Yuan Y, Wang X. Identification of differentially expressed genes between triple and non-triple-negative breast cancer using bioinformatics analysis. Breast Cancer. 2019;26(6):784-91.

3. Mouh FZ, Mzibri ME, Slaoui M, Amrani M. Recent Progress in triple negative breast cancer research. Asian Pac J Cancer Prev. 2016;17(4):1595-608.

4. Reddy GM, Suresh PK, Pai RR. Clinicopathological features of triple negative breast carcinoma. J Clin Diagn Res. 2017;1 1(1):Ec05-8.

5. Thakur V, Kutty RV. Recent advances in nanotheranostics for triple negative breast cancer treatment. J Exp Clin Cancer Res. 2019;38(1):430.

6. Perez DS, Hoage TR, Pritchett JR, Ducharme-Smith AL, Halling ML, Ganapathiraju SC, et al. Long, abundantly expressed non-coding transcripts are altered in cancer. Hum Mol Genet. 2008;17(5):642-55.

7. Guttman M, Donaghey J, Carey BW, Garber M, Grenier JK, Munson G, et al. lincRNAs act in the circuitry controlling pluripotency and differentiation. Nature. 2011;477(7364):295-300.

8. Ponting CP, Oliver PL, Reik W. Evolution and functions of long noncoding RNAs. Cell. 2009;136(4):629-41. 
9. Xiang J, Guo S, Jiang S, Xu Y, Li J, Li L, et al. Silencing of long non-coding RNA MALAT1 promotes apoptosis of glioma cells. J Korean Med Sci. 2016;31(5):688-94.

10. Evans JR, Feng FY, Chinnaiyan AM. The bright side of dark matter: IncRNAs in cancer. J Clin Investig. 2016;126(8):2775-82.

11. Gibb EA, Vucic EA, Enfield KS, Stewart GL, Lonergan KM, Kennett JY, et al. Human cancer long non-coding RNA transcriptomes. PloS ONE. 2011;6(10): e25915.

12. Moran VA, Perera RJ, Khalil AM. Emerging functional and mechanistic paradigms of mammalian long non-coding RNAs. Nucleic Acids Res. 2012:40(14):6391-400.

13. Shin VY, Chen J, Cheuk IW, Siu MT, Ho CW, Wang X, et al. Long non-coding RNA NEAT1 confers oncogenic role in triple-negative breast cancer through modulating chemoresistance and cancer stemness. Cell Death Dis. 2019;10(4):270.

14. Gooding AJ, Zhang B, Gunawardane L, Beard A, Valadkhan S, Schiemann WP. The IncRNA BORG facilitates the survival and chemoresistance of triple-negative breast cancers. Oncogene. 2019:38(12):2020-41.

15. Luo L, Tang H, Ling L, Li N, Jia X, Zhang Z, et al. LINC01638 IncRNA activates MTDH-Twist1 signaling by preventing SPOP-mediated c-Myc degradation in triple-negative breast cancer. Oncogene. 2018;37(47):6166-79.

16. Kopp F, Mendell JT. Functional classification and experimental dissection of long noncoding RNAs. Cell. 2018;172(3):393-407.

17. Wang YG, Wang T, Shi M, Zhai B. Long noncoding RNA EPB41L4A-AS2 inhibits hepatocellular carcinoma development by sponging miR301a-5p and targeting FOXL1. J Exp Clin Cancer Res. 2019;38(1):153.

18. Huo H, Tian J, Wang R, Li Y, Qu C, Wang N. Long non-coding RNA NORAD upregulate SIP1 expression to promote cell proliferation and invasion in cervical cancer. Biomed Pharmacother. 2018:106:1454-60.

19. Zou Z, Ma T, He X, Zhou J, Ma H, Xie M, et al. Long intergenic non-coding RNA 00324 promotes gastric cancer cell proliferation via binding with HuR and stabilizing FAM83B expression. Cell Death Dis. 2018;9(7):717.

20. Yang Z, Dong X, Pu M, Yang H, Chang W, Ji F, et al. LBX2-AS1/miR-219a2-3p/FUS/LBX2 positive feedback loop contributes to the proliferation of gastric cancer. Gastric Cancer . 2020;23(3):449-63.

21. Sun J, Zhang Y, Li B, Dong Y, Sun C, Zhang F, et al. PITPNA-AS1 abrogates the inhibition of miR-876-5p on WNT5A to facilitate hepatocellular carcinoma progression. Cell Death Dis. 2019;10(11):844.

22. Livak KJ, Schmittgen TD. Analysis of relative gene expression data using real-time quantitative PCR and the 2(-Delta Delta C(T)) Method. Methods. 2001;25(4):402-8.

23. Feng $\mathrm{H}$, Wei B, Zhang Y. Long non-coding RNA HULC promotes proliferation, migration and invasion of pancreatic cancer cells by down-regulating microRNA-15a. Int J Biol Macromol. 2019;126:891-8.

24. Wen D, Huang Z, Li Z, Tang X, Wen X, Liu J, et al. LINC02535 co-functions with $P C B P 2$ to regulate DNA damage repair in cervical cancer by stabilizing RRM1 mRNA. J Cell Physiol. 2020;235(10):7592-603.

25. Tang J, Zhong G, Zhang H, Yu B, Wei F, Luo L, et al. LncRNA DANCR upregulates PI3K/AKT signaling through activating serine phosphorylation of RXRA. Cell Death Dis. 2018;9(12):1167.
26. Wang B, Lu FY, Shi RH, Feng YD, Zhao XD, Lu ZP, et al. MiR-26b regulates 5-FU-resistance in human colorectal cancer via down-regulation of Pgp. Am J Cancer Res. 2018:8(12):2518-27.

27. Zhou Y, Ren H, Dai B, Li J, Shang L, Huang J, et al. Hepatocellular carcinoma-derived exosomal miRNA-21 contributes to tumor progression by converting hepatocyte stellate cells to cancer-associated fibroblasts. J Exp Clin Cancer Res. 2018;37(1):324.

28. Zhang Y, Yang G, Luo Y. Long non-coding RNA PVT1 promotes glioma cell proliferation and invasion by targeting miR-200a. Exp Ther Med. 2019;17(2):1337-45

29. Gao J, Liu L, Li G, Cai M, Tan C, Han X, et al. LncRNA GAS5 confers the radio sensitivity of cervical cancer cells via regulating miR-106b/IER3 axis. Int J Biol Macromol. 2019:126:994-1001.

30. Zhi T, Jiang K, Xu X, Yu T, Wu W, Nie E, et al. MicroRNA-520d-5p inhibits human glioma cell proliferation and induces cell cycle arrest by directly targeting PTTG1. Am J Transl Res. 2017;9(11):4872-87.

31. Li T, Guo H, Zhao X, Jin J, Zhang L, Li H, et al. Gastric cancer cell proliferation and survival is enabled by a cyclophilin B/STAT3/miR-520d-5p signaling feedback loop. Can Res. 2017;77(5):1227-40.

32. Yan L, Yu J, Tan F, Ye GT, Shen ZY, Liu H, et al. SP1-mediated microRNA520d-5p suppresses tumor growth and metastasis in colorectal cancer by targeting CTHRC1. Am J Cancer Res. 2015;5(4):1447-59.

33. Zhao J, Zhang X, Gao T, Wang S, Hou Y, Yuan P, et al. SIK2 enhances synthesis of fatty acid and cholesterol in ovarian cancer cells and tumor growth through PI3K/Akt signaling pathway. Cell Death Dis. 2020;11(1):25.

34. Maxfield KE, Macion J, Vankayalapati H, Whitehurst AW. SIK2 restricts autophagic flux to support triple-negative breast cancer survival. Mol Cell Biol. 2016;36(24):3048-57.

35. Zhang Y, Guo H, Zhang H. SNHG10/DDX54/PBX3 feedback loop contributes to gastric cancer cell growth. Dig Dis Sci. 2020. https://doi.org/10. 1007/s 10620-020-06488-9.

36. Milek M, Imami K, Mukherjee N, Bortoli F, Zinnall U, Hazapis O, et al. DDX54 regulates transcriptome dynamics during DNA damage response. Genome Res. 2017;27(8):1344-59.

37. Qiao K, Ning S, Wan L, Wu H, Wang Q, Zhang X, et al. LINC00673 is activated by $Y Y 1$ and promotes the proliferation of breast cancer cells via the miR-515-5p/MARK4/Hippo signaling pathway. J Exp Clin Cancer Res . 2019;38(1):418.

38. Tran DDH, Kessler C, Niehus SE, Mahnkopf M, Koch A, Tamura T. Myc target gene, long intergenic noncoding RNA, Linc00176 in hepatocellular carcinoma regulates cell cycle and cell survival by titrating tumor suppressor microRNAs. Oncogene. 2018;37(1):75-85.

39. Liu C, Zhang YH, Huang T, Cai Y. Identification of transcription factors that may reprogram lung adenocarcinoma. Artif Intell Med. 2017;83:52-7.

\section{Publisher's Note}

Springer Nature remains neutral with regard to jurisdictional claims in published maps and institutional affiliations.
Ready to submit your research? Choose BMC and benefit from:

- fast, convenient online submission

- thorough peer review by experienced researchers in your field

- rapid publication on acceptance

- support for research data, including large and complex data types

- gold Open Access which fosters wider collaboration and increased citations

- maximum visibility for your research: over 100M website views per year

At BMC, research is always in progress.

Learn more biomedcentral.com/submissions 